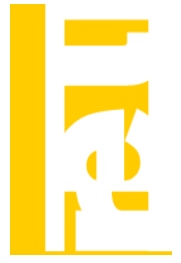

\title{
CONCILIACIÓN ENTRE VIDA LABORAL Y CUIDADOS INFORMALES A PERSONAS MAYORES DEPENDIENTES EN ESPAÑA
}

\author{
Cristina Vilaplana*1 \\ *Universidad de Murcia
}

Recibido el 25 de septiembre de 2009; aceptado el 3 de diciembre de 2010

\section{Resumen}

En este trabajo se analiza la participación laboral de los cuidadores de personas mayores utilizando la Encuesta de Apoyo Informal (2004). Se estima un modelo probit ordenado para la incidencia de problemas laborales considerando la existencia de sesgo de selección por el hecho de estar ocupado antes de ser cuidador, y que las horas de cuidado informal y las necesidades no satisfechas de atención formal son variables endógenas. Los hombres y los más jóvenes son menos propensos a sufrir problemas laborales, mientras que las mujeres, los cuidadores de mayor antigüedad, los cuidadores de grandes dependientes o residentes en municipios pequeños manifiestan mayor probabilidad de dejar su ocupación. No obstante, la aparición de problemas es independiente del número de años de cuidado a partir de una carga de 3 a 5 horas diarias, en parte debido a que el cuidador trata de suplir las carencias de atención formal sacrificando su vida laboral.

Palabras clave: Cuidados informales, cuidador, mercado de trabajo, dependencia Clasificación JEL: J14, J22.

\begin{abstract}
This paper analyses labour participation of informal caregivers of elder people using the Informal Support Survey (2004). We estimate an ordered probit model for the incidence of labour problems considering the existence of sample selection due to working status before becoming caregiver and that informal caregiving hours and formal care unmet needs are endogenous variables. Men and youngest people are less prone of suffering labour problems, whereas women, caregivers with more caregiving years, caregivers of great dependent people or those living in small municipalities attain a higher probability of quitting their job. However, the emergence of problems is independent from the number of caregiving years for a load between 3 and 5 daily caregiving hours, in part due to the fact that the informal caregiver tries to substitute formal care deficiencies giving up his personal working life.
\end{abstract}

Key words: Informal care, caregiver, labour market, dependency.

JEL Classification: J14, J22.

\footnotetext{
1 Dirección para correspondencia: cvilaplana@um.es. La autora desea hacer constar su agradecimiento a un revisor anónimo por sus comentarios y sugerencias que han contribuido a mejorar la calidad del artículo.
}

(C) Revista de Economía Laboral 


\section{Introducción}

Tradicionalmente, la familia ha constituido el principal proveedor de cuidados a las personas mayores dependientes. Sin embargo, los cambios en las pautas demográficas y socioeconómicas pueden debilitar el papel de la misma como pilar fundamental de los cuidados a las personas mayores. Por el lado de la demanda de cuidados, la disminución en las tasas de mortalidad ha convertido a la cohorte de mayores de 65 años en la de más rápido crecimiento de la población española. Por otra parte, a pesar del aumento en los años de vida libres de discapacidad (67,7 en 1996 a 70,2 en 2003), el número de personas de 65 y más años que necesita ayuda para realizar tareas de la vida diaria se ha incrementado, pasando de 2.072.600 en 1999 a 2.227.500 en $2008^{2}$.

Por el lado de la oferta de cuidadores, hay que tener en cuenta una serie de factores como la disminución en la tasa de fecundidad, el aumento en la tasa de divorcios y la creciente participación femenina en el mercado de trabajo ${ }^{3}$, que supondrán dentro de unos años, bien una reducción en el número de cuidadores potenciales, bien una disminución en la capacidad de uno de los cónyuges para cuidar del otro, o en la cantidad de tiempo del que puede disponer para ejercer el papel de cuidador.

De hecho, las responsabilidades asociadas al trabajo pueden llegar a ser incompatibles con las exigencias que conlleva ser cuidador. De acuerdo con la información del Módulo Especial de Conciliación entre Vida Familiar y Laboral (Encuesta de Población Activa, 2005), un 12,93\% (16,21\%) de los hombres (mujeres) ocupados(as) que cuidaban a niños y/o dependientes desearía trabajar menos para aumentar el tiempo de cuidado dedicado a estas personas. Cuando se preguntaba a las cuidadores de personas dependientes que habían reducido su jornada laboral el motivo por el que habían tomado esa decisión, un 31,69\% (42,37\%) de los cuidadores(as) afirmaba que se debía a la falta de servicios de atención para personas dependientes y un 51,23\% (46,11\%) opinaba que los servicios disponibles estaban fuera de sus posibilidades económicas. Finalmente, de entre aquellos(as) cuidadores(as) que habían comentado la falta de servicios para personas mayores, un 51,64\% (40,04\%) puntualizó

\footnotetext{
2 Tablas de mortalidad (INE) y Public Health \& Structural Indicators on Health (Eurostat)..

${ }^{3}$ El número medio de hijos por mujer ha descendido de 2,803 en 1975 a 1,458 en 2008; la tasa de divorcios por 100 matrimonios ha subido de $4,7 \%$ en 1989 a $63,92 \%$ en 2006 y la tasa de participación femenina ha subido de $31,83 \%$ en 1987 a 51,55\% en 2009. (Indicadores Demográficos Básicos; Indicadores Sociales; Encuesta de Población Activa; INE).
} 
que la demanda no satisfecha de atención formal se concentraba entre las 7 y las 19 horas (horario laboral) mientras que un 40,56\% (44,15\%) manifestaba el deseo de recibir estos servicios también en horarios especiales (noche y fines de semana).

La evidencia más flagrante de que cuidados informales y trabajo pueden resultar dos facetas imposibles de reconciliar se obtiene al observar las tasas de participación de los cuidadores de entre 25 y 64 años: $69,99 \%$ para los hombres y $38,74 \%$ para las mujeres (Encuesta de Apoyo Informal, 2004). Hasta qué punto las transferencias de tiempo del cuidador a la persona dependiente pueden conllevar la aparición de problemas en el trabajo, o incluso hacer incompatible la conciliación de ambas facetas es el objetivo de este trabajo.

Se propone estimar un modelo para los problemas laborales (probit ordenado) con dos variables potencialmente endógenas (horas informales de cuidado (continua) y necesidades no satisfechas (probit)). El fundamento es que el hecho de tener problemas laborales puede repercutir en la cantidad de cuidados prestados, pero también a mayor número de horas de cuidado más dificultades para conciliar la faceta de cuidador y de trabajador. Por otra parte, el hecho de no recibir una adecuada atención formal (necesidad no satisfecha) puede generar en el cuidador el sentimiento de que él debe responsabilizarse de esa carencia, y consecuentemente, al incrementarse la carga de cuidados tenderían a aumentar los problemas laborales. Pero al mismo tiempo, si el cuidador tiene problemas laborales puede aumentar la propensión a solicitar ayudas formales, y en este contexto se produciría un aumento de las posibilidades de rechazo, o incluso, de que recibiendo dicho servicio formal, no lo considerase suficientemente útil. En este modelo además se tiene en cuenta (i) el posible sesgo de selección ya que la muestra de cuidadores está escogida de entre aquellos cuidadores que estaban ocupados en el momento de empezar a prestar cuidados y (ii) la correlación entre variables inobservables, puesto que se estima explícitamente la matriz de correlación entre los residuos.

Para ello se va a utilizar la Encuesta de Apoyo Informal (2004) que contiene información detallada de cuidadores de personas mayores de 60 años, aunque también presenta ciertas limitaciones. Primero, no conocemos si el individuo se convirtió en cuidador porque la persona dependiente sufría un problema de necesidades no satisfechas o si esta situación se originó una vez empezados los cuidados informales. Segundo, ignoramos si el cuidador decidió adaptar su horario de trabajo debido a las exigencias de los cuidados desde el momento en que asumió dichas funciones de cuidador o si intentó mantener su vida laboral hasta que le fue imposible compaginarla con las labores de cuidador. Tercero, en el caso 
de cuidadores que ya no trabajan y además sufren necesidades no satisfechas desconocemos si ése fue el desencadenante de que decidiera dejar de trabajar.

Las principales contribuciones del trabajo son las siguientes: (i) los perjuicios laborales soportados por la mujeres son mayores independientemente de cuál sea la relación de parentesco con la persona receptora de cuidados, (ii) cuanto menor sea la edad del cuidador en términos absolutos o respecto a la persona dependiente mayor es la probabilidad de continuar trabajando sin problemas, (iii) los empresarios sin asalariados y los trabajadores sin cualificar son más vulnerables a sufrir problemas laborales respecto a las otras categorías profesionales, (iv) conforme aumenta la antigüedad del cuidador aumenta la probabilidad de dejar el mercado de trabajo, pero la antigüedad no es relevante para la probabilidad de sufrir problemas laborales cuando se considera una carga a partir de "3-5 horas/día", (v) el cuidado de enfermos mentales, grandes dependientes o la carencia de la adecuada atención formal conllevan un fuerte aumento de la probabilidad dejar el trabajo, (vi) a medida que aumenta el tamaño del municipio de residencia se incrementa la probabilidad de no tener problemas laborales y, en menor medida, se reduce la probabilidad de dejar el empleo y (vii) en presencia de necesidades no satisfechas de atención formal, los cuidadores informales aumentan su dedicación para suplir dichas deficiencias de calidad o cantidad, contradiciendo por tanto la teoría de sustitución entre cuidados formales e informales.

El artículo se organiza como sigue. En la sección 2 se realiza una revisión de la literatura. En la sección 3 se plantea el modelo econométrico y en la sección 4 se describe la muestra utilizada. En la sección 5 se discuten las estimaciones y predicciones obtenidas, y por último, en la sección 6 se comentan las implicaciones de política social y laboral.

\section{Revisión de la literatura sobre cuidados informales y mercado de trabajo}

A menudo se ha considerado que los cuidados informales constituyen una fuente de ayuda gratuita, cuando en realidad implican un importante coste de oportunidad soportado por el cuidador: deterioro de la salud, disminución del tiempo de ocio (vacaciones, vida social), reducción de la atención dispensada al cónyuge e hijos, aumento de los gastos corrientes y problemas en el trabajo (Baldwin, 1985; Pearlin et al, 1990; Gallager y Mechanic, 1996). 
Este trabajo se concentra en esta última categoría de costes. A corto plazo, las tareas relativas al cuidado compiten con el empleo y pueden ocasionar pérdidas de horas de trabajo y de ingresos. A lo largo del tiempo, estos efectos negativos se pueden acumular, limitando el acceso del cuidador a una pensión de jubilación o reduciendo la cuantía de la misma, lo que en último término puede incrementar el riesgo de situarse por debajo del umbral de pobreza. Por otra parte, desde un punto de vista agregado, la pérdida de trabajadores afectará negativamente a la productividad de la economía, y en el contexto de unas sociedades envejecidas disminuirá el ratio entre activos y pasivos. Existen numerosos estudios que han analizado la relación entre cuidados informales y oferta de trabajo, llegando a conclusiones diversas.

En relación a los trabajos que han utilizado datos de sección cruzada, algunos han encontrado una relación negativa entre trabajo y cuidados informales (Stone et al. 1987; Dwyer y Coward, 1991; Boaz y Muller, 1992), mientras que otros no han hallado evidencias significativas de que exista un trade-off entre ambas variables (Wolf y Soldo, 1994; Stern, 1995; Ettner, 1996). Esta falta de homogeneidad en los resultados ha sido atribuida a la modelización en forma reducida utilizada, puesto que unos autores han considerado la decisión de proporcionar cuidados como variable dependiente y la oferta de trabajo como variable explicativa, mientras que otros sostienen que la decisión de trabajar y las horas de trabajo son una función de los cuidados informales. Pero dado que en estos modelos la decisión de proveer cuidados informales y de trabajar se realizan de manera simultánea, no se puede inferir causalidad, ya que no se puede asegurar si los cuidadores proporcionan cuidados porque trabajan menos horas o, por el contrario, trabajan menos horas debido a la carga que soportan.

En relación a los trabajos con datos de panel, la mayoría de los trabajos se han concentrado en Estados Unidos y en el Reino Unido. En el primer caso, McGarry (2003), a partir del Health and Retirement Study, halló una relación muy débil entre la relación con la actividad económica antes de convertirse en cuidador y los cuidados informales dispensados llegado el momento, tanto en el corto como en el largo plazo. En cambio, Pavalko y Artis (1997) con datos del National Longitudinal Survey of Mature Women observaron que las mujeres que habían empezado a ser cuidadoras reportaban un número de horas de trabajo significativamente inferior respecto a otras que nunca habían sido cuidadoras. En un trabajo posterior (Pavalko y Henderson, 2006) evidenciaron que el hecho de tener un horario flexible o poder solicitar una baja por motivos familiares, aunque sin sueldo, aumentaba la probabilidad de permanencia en el trabajo. Doty et al. (1998), con datos del National Long Term Care Survey, 
Vilaplana / Revista de Economía Laboral 7 (2010), 85-125

y Kolodinski y Shirey (2000), a partir del Panel Study of Income Dynamics, llegaron a las mismas conclusiones, aunque en el último caso sólo para el caso de cuidadoras co-residentes con personas dependientes. Para el Reino Unido, Carmichael y Charles (1998, 2003), a partir del British Household Panel Study, constataron que el hecho de prestar cuidados afectaba negativamente a la oferta de trabajo y a los ingresos salariales. En cambio, Heitmueller y Michaud (2006) analizaron el mismo panel, pero concluyeron que el hecho de ser cuidador reducía la probabilidad de estar trabajando sólo en el caso de que el cuidador y el dependiente fuesen co-residentes. De todas formas, hay que precisar que en este análisis no se introdujo información sobre la intensidad de los cuidados dispensados.

En relación a la evidencia existente para España, hay que remitirse a los trabajos que han utilizado encuestas europeas y en los cuales España suele aparecer incluida dentro de los "países del Sur" junto con Italia y Grecia. Existen numerosos estudios que se han basado en el European Community Household Panel. En el trabajo de Spiess y Schneider (2002) se demuestra que existe una relación significativa y negativa entre el inicio de las funciones como cuidador y los cambios en el número de horas semanales trabajadas, remarcando además que es poco probable recuperar los niveles iniciales de participación e intensidad una vez finalizado el periodo de cuidados. Por su parte, Viitanen (2005) introdujo algunos aspectos que no habían sido tomados en consideración en el trabajo de Spiess y Schneider: efectos específicos por país, heterogeneidad inobservable y dependencia de estado. Sus resultados indicaban que las mujeres de mediana edad eran las más afectadas cuando se convertían en cuidadoras, presentando una menor tasa de participación hasta la edad de retiro. Además, las mujeres solteras cuidadoras eran más propensas a encontrarse por debajo del umbral de pobreza en su vejez debido a una menor inserción en el mercado de trabajo. Estos resultados eran particularmente significativos en Alemania, Grecia, Italia y Países Bajos.

Con esta misma encuesta, es preciso resaltar el trabajo de Casado et al. (2010) en el que se constata una reducción en la probabilidad de estar ocupadas entre las cuidadoras co-residentes con la persona dependiente y entre las cuidadoras que proporcionaban cuidados durante más de un periodo. Sin embargo, no detectaron problemas para reincorporararse al mercado de trabajo en el caso de las cuidadoras que habían finalizado la prestación de cuidados informales. El trabajo de Casado et al. (2010) presenta dos importantes ventajas: (1) permite comparar la participación en el mercado de trabajo (jornada completa o parcial) entre cuidadoras y no cuidadoras, y (2) es posible relacionar el 
momento de salida del mercado de trabajo con el número de años de cuidado. En cambio, con la Encuesta de Apoyo Informal (2004), que se utilizará en este trabajo, todos los individuos entrevistados son cuidadores, y aunque se conoce si tienen problemas laborales y el número de años que llevan cuidando al dependiente, no es posible determinar cuándo empezaron dichos problemas. Como contrapartida, el presente artículo analiza el impacto de nuevas variables potencialmente relevantes sobre la incidencia de problemas laborales: grado y nivel de dependencia del receptor de cuidados, patologías padecidas, situación profesional del cuidador, características de los servicios sociales para dependientes y tamaño del municipio de residencia.

Otra fuente de información es el Survey of Health, Ageing and Retirement in Europe (SHARE). Utilizando esta encuesta, Crespo (2008) ha analizado la participación en el mercado de trabajo de mujeres de mediana edad que cuidan de alguno de sus progenitores, concluyendo que existe una relación significativa y negativa entre ambas variables, siendo dicha evidencia más intensa cuando los cuidados informales son considerados como una variable endógena en la ecuación de participación.

En el presente trabajo sobre los problemas laborales de los cuidadores españoles, se ha optado por utilizar la Encuesta de Apoyo Informal (2004). Existe un claro trade-off entre estudiar la decisión de convertirse en cuidador, para lo cual serían apropiadas el PHOGUE y el SHARE, y analizar las repercusiones que la carga de cuidados tiene sobre la vida laboral del cuidador, en cuyo caso, habría que inclinarse por la Encuesta de Apoyo Informal. En primer lugar, en el PHOGUE (2001) se dispone de información de 464 cuidadores informales de entre 25-64 años, de los cuales 339 sólo cuidan a personas mayores y 125 que además cuidan niños. Dado que las preguntas sobre la existencia de problemas laborales son del tipo "el hecho de cuidar niños u otras personas le impide llevar a cabo la cantidad o el tipo de trabajo que de otras formas realizaría" o "la principal razón para no buscar trabajo es el cuidado de hijos u otras personas", para estas 125 personas no se puede determinar si los problemas laborales, en caso de padecerlos, se deben exclusivamente al cuidado de adultos dependientes. Respecto al SHARE, no es posible realizar una comparación directa con la Encuesta de Apoyo Informal, puesto que la primera sólo es representativa de cuidadores de 50 años en adelante, y si aparecen datos de individuos más jóvenes es debido a que también son entrevistadas las parejas de los seleccionados. En cambio, los cuidadores entrevistados en la Encuesta de Apoyo Informal tienen 16 y más años.

A efectos de ampliar las posibilidades de comparación, se ha recurrido a otra encuesta a nivel internacional e incluso más actual que el 
SHARE Eurobarómetro 67.3 sobre "Health and Long Term Care in the European Union" (European Comisión 2007) ${ }^{4}$. Se trata de una encuesta llevada a cabo en la Unión Europea-27, Croacia y Turquía. Se entrevistó a 28.660 personas de 16 y más años, de las cuales la muestra correspondiente a España es de 1.007 observaciones. Al analizar los datos, cuando se pregunta al entrevistado si ha prestado apoyo a otra persona que requiriera cuidados de largo plazo, se ha considerado que el entrevistado era cuidador informal si respondía afirmativamente a alguna de las siguientes tareas "preparar comidas, limpiar la casa, hacer la compra, ayudar a dar de comer, a vestir, a moverse, a ir al aseo, a lavarse". A continuación, se pregunta si por causa de ser cuidador informal, el entrevistado tuvo que dejar de trabajar. Para el caso de España, un total de 292 deberían ser consideradas cuidadoras, de las cuales 48 dejaron de trabajar total o parcialmente por motivos de las exigencias de los cuidados. Por tanto, la muestra disponible con la Encuesta de Apoyo Informal, aunque no es tan actual como la del Eurobarómetro 67.3, sí es más mucho más amplia (con 1.137 observaciones de cuidadores de los que 675 tienen algún problema laboral) y proporciona una buena base para realizar este estudio.

\section{Modelo econométrico}

\subsection{Modelo probit ordenado con sesgo de selección y dos variables endógenas}

El modelo propuesto pretende contrastar el efecto de las necesidades no satisfechas y de las horas de cuidado sobre la probabilidad de tener problemas laborales. El fundamento para relacionar estas tres variables es que si cuando una persona dependiente no institucionalizada sufre una necesidad no satisfecha de atención formal ${ }^{5}$ (Atención a

\footnotetext{
${ }^{4}$ http://ec.europa.eu/public_opinion/archives/ebs/ebs_283_en.pdf

5 Se ha optado por la variable "necesidades no satisfechas" en lugar de "recibir ayudas formales" porque se considera que lo verdaderamente relevante a la hora de determinar el impacto de los servicios formales para personas dependientes sobre la vida laboral del cuidador es la diferencia entre el bienestar esperado (en caso de solicitarlas) y el bienestar percibido. Bajo la variable binaria "recibir ayudas formales" se pueden estar agregando realidades muy diferentes: dentro de la categoría "uno" se incluye a los individuos que reciben y están satisfechos con el servicio, y a los que reciben, pero consideran que sería mejorable, y dentro de la categoría "cero" se incluye la situación de los individuos que no reciben este servicio, porque no han creído conveniente solicitarlo, y la de los individuos que lo solicitaron, pero no lo han recibido. Por el contrario, cada una de las categorías de la variable "necesidades no satisfechas" representa la
} 
Domicilio, Centro de Día), entonces uno de los miembros de la familia reacciona proporcionando la cantidad requerida de cuidados, los cuidados informales estarán actuando como un "colchón" que alivie las deficiencias de los cuidados formales. En estas circunstancias, es posible que el cuidador deba reorganizar su vida familiar y laboral, debiendo incluso renunciar a parte de ella.

Por consiguiente, se va a estimar un modelo compuesto por tres ecuaciones simultáneas (problemas laborales, horas de cuidado y necesidades no satisfechas) y dos variables potencialmente endógenas (necesidades no satisfechas y horas de cuidados informales en la ecuación para modelizar los problemas laborales, y necesidades no satisfechas en la ecuación para horas de cuidado informal). Adicionalmente, se tiene en cuenta la existencia de un sesgo de selección motivado por el hecho de que sólo pueden sufrir problemas laborales aquellos cuidadores que previamente se encuentran trabajando.

Este tipo de modelo simultáneo requiere una condición de coherencia que viene impuesta por su forma triangular (Maddala, 1983; Blundell y Smith, 1994) ${ }^{6}$. Dicha condición de coherencia implica que la variable "problemas laborales" no puede introducirse en ninguna de las otras dos ecuaciones, así como tampoco la variable "horas de cuidado informal' puede introducirse en la ecuación para modelizar las "necesidades no satisfechas".

Es evidente que las "horas de cuidado informal" pueden afectar a las necesidades no satisfechas (por ejemplo, un mayor número de horas afectará a la probabilidad de solicitar ayuda formal, y por consiguiente, a

agregación de realidades homogéneas: si toma el valor "uno" es porque el individuo considera que se ha producido una ineficiencia en el proceso de asignación de recursos (o bien no lo recibe, o lo recibe en cantidad insuficiente, o no está conforme con la calidad del mismo), y si toma el valor "cero" es porque el individuo está conforme con su situación (o lo recibe y está contento con la calidad o cantidad del mismo, o bien no lo recibe porque no lo solicitó, aunque declara conocer la existencia del mismo).

${ }^{6}$ El planteamiento econométrico de este modelo es acorde con el de Meyerhoefer and Pylypchuck (2008) que estiman un probit ordenado para el índice de masa corporal considerando como variables endógenas el gasto sanitario (continua) y la participación en el programa de bonos para comprar comida (discreta) y con el trabajo de Mroz y Guilkey (1992) que llevan a cabo una serie de experimentos de Monte Carlo para un modelo con una variable dependiente discreta (probit) y dos variables endógenas (continua y discreta) y sesgo de selección. A diferencia del primer modelo, en nuestro caso no utilizamos datos de panel, sino una sección cruzada, pero introducimos un sesgo de selección, y con respecto al segundo, nuestra variable dependiente es un probit ordenado. Respecto a la interpretación de coeficientes obtenidos a partir de aproximaciones discretas, Mroz y Guilkey (1992) manifiestan la fiabilidad de las estimaciones obtenidas y su utilidad para la investigación empírica. 
la probabilidad de que surjan necesidades no satisfechas). Para tener en cuenta estas interrelaciones, satisfaciendo al mismo tiempo la condición de coherencia, es obligatorio incluir todas las variables exógenas de la ecuación de "problemas laborales" en las otras dos ecuaciones. De esta forma se puede apreciar el efecto de las características observables que determinan la probabilidad de sufrir problemas laborales, tanto sobre las horas de cuidado informal como sobre las necesidades no satisfechas. De la misma manera, todas las variables que afectan a la variable "horas de cuidado informal" se deben incluir en la ecuación para las "necesidades no satisfechas" para así recoger el efecto de dedicar más horas sobre la aparición de las mismas.

Por consiguiente, este modelo simultáneo puede considerarse como un modelo en el que existe una ecuación de interés (probabilidad de tener problemas laborales) y las otras dos ecuaciones no son más que formas reducidas. Desde este punto de vista lo que es importante para que las ecuaciones de horas de cuidado informal y necesidades no satisfechas estén identificadas apropiadamente, es demostrar que existen restricciones de exclusión relevantes (véase sección 5.1 para test de diagnóstico).

Aunque la estrategia de estimación planteada permite tratar los problemas de endogeneidad de las horas de cuidado y las necesidades no satisfechas, pudiera ser que las estimaciones sufrieran otros sesgos aleatorios. Por ejemplo, shocks aleatorios comunes a todos los individuos sujetos a la misma política regional de servicios sociales a la dependencia, en cuyo caso se generarían efectos correlacionados. Para evitar este problema se han realizado clusters por comunidades autónomas y se han obtenido los errores estándar ajustados para tener en cuenta la dependencia potencial de los residuos entre regiones (Wooldrige, 2003) ${ }^{7}$.

En definitiva, sean $P L, H I$ y $N N S$ tres variables que denotan la existencia de problemas laborales derivados de las implicaciones como cuidador informal, las horas diarias de cuidados informales y la existencia de necesidades no satisfechas,

\footnotetext{
7 En las estimaciones se incluye la tasa de cobertura del servicio Atención a Domicilio y de los Centros de Día. La tasa de cobertura se define como el número de usuarios dividido por la población de 65 y más años. También se incluye el porcentaje del tiempo de Atención a Domicilio dedicado a cuidados personales. De todas formas, hay otras variables de política regional que no se controlan, como por ejemplo la forma en la que se concedían las ayudas formales en el año 2004, ya que cada comunidad autónoma utilizaba una tabla de pesos diferente para las variables: discapacidad física, discapacidad mental, recursos financieros, habitabilidad de la vivienda, disponibilidad de la familia. (Véase páginas 96 y 97 de Atención a las personas en situación de dependencia en España. Libro Blanco (2004)).
} 


$$
\begin{aligned}
& P L_{i}=\alpha_{1} \Pi_{1 i}+\kappa_{1} H I_{i}+\kappa_{2} N N S+\varepsilon_{1 i} \\
& H I_{i}=\alpha_{2} \Pi_{2 i}+\kappa_{3} N N S+\varepsilon_{2 i} \\
& N N S_{i}=\alpha_{3} \Pi_{3 i}+\varepsilon_{3 i}
\end{aligned}
$$

donde $\Pi_{1}$ es un vector de variables exógenas referidas a la persona dependiente (edad, sexo, patologías, grado de dependencia, vive solo) y al cuidador (edad, sexo, estudios, situación profesional, sustentador principal, número de años prestando cuidados) ${ }^{8} ; \Pi_{2}$ incluye las mismas variables que $\Pi_{1}$ y además relación de parentesco entre dependiente y cuidador, diferencia de edad y diferencia de género ${ }^{9} ; \Pi_{3}$ incluye las mismas variables que $\Pi_{2}$ y además ingresos mensuales del dependiente, tamaño del municipio de residencia, tasa de cobertura del Servicio de Atención a Domicilio y de Centros de Día y porcentaje del tiempo de Atención a Domicilio dedicado a cuidados personales.

El hecho de incluir la diferencia de sexo como restricción de exclusión se debe a que entre los factores que influyen en las preferencias del receptor de cuidados figuran factores emocionales (relación de afecto), pero también culturales (por ejemplo, para determinados cuidados íntimos la persona dependiente puede preferir a un cuidador del mismo sexo). Aunque los datos no incluyen referencias sobre las preferencias del receptor de cuidados, es posible contrastar esta hipótesis de forma indirecta mediante la definición de una variable binaria para la coincidencia de sexos entre cuidador y dependiente. La literatura que ha explorado este tema no es concluyente al respecto, Algunos estudios (Stoller, 1983; Pillemer y Suitor, 2006) encontraron evidencia a favor de lo que se ha denominado "cross-sex taboo", mientras que los resultados de otros (Howorwitz, 1985; Arber y Ginn, 1995) no apoyaban esta hipótesis. Por tanto, no sólo la relación de parentesco, sino también la diferencia (coincidencia) de sexo puede ser una variable significativa para explicar la

\footnotetext{
${ }^{8}$ La imposición de restricciones de exclusión no es absolutamente obligatoria puesto que el modelo está identificado a través de la forma funcional (Heckman, 1978). Por si dicha identificación resultara feble se han introducido algunas restricciones de identificación siguiendo un procedimiento de lo general a lo particular. Se comenzó especificando las variables que se consideraron razonables para explicar los problemas laborales de los cuidadores (según la revisión de la literatura de la sección 2). A continuación, se llevaron a cabo test de exclusión de Wald para variables pertenecientes a la misma categoría. Se mantuvieron aquellos grupos de variables que eran significativos al 5\%. Por ejemplo, en la especificación inicial para los problemas laborales se incluyeron también estado civil, tamaño del municipio de residencia e hijos presentes en el hogar, pero dichos grupos de variables no resultaron significativos al $5 \%$.

${ }^{9}$ La diferencia de edad se calcula como edad del dependiente menos edad del cuidador y la diferencia de género es una variable binaria que toma el valor uno cuando cuidador y dependiente son del mismo sexo.
} 
carga en término de tareas (y por consiguiente de horas) soportada por los cuidadores.

La diferencia de edad entre el dependiente y el cuidador también es otra variable reveladora de las preferencias de la persona receptora de cuidados. En caso de resultar significativa y negativa, indicaría que cuanto menor sea la diferencia de edad entre ambos, más probable es el hecho de ser cuidador (Engers y Stern, 2002). Si bien por un lado, liberaría de carga a los "cuidadores potenciales" más jóvenes (y favorecería su permanencia en el mercado laboral), por otro lado, implicaría que el cuidador también es una persona de edad avanzada, aumentando la probabilidad de que ellos mismos padezcan también discapacidades. Si por el contrario el coeficiente de la diferencia de edad es significativo y positivo, supondría que los cuidadores más jóvenes tienen más probabilidad de estar implicados en las tareas del cuidado, lo que puede derivar en un problema de conciliación entre vida laboral y familiar. Precisamente, en el trabajo de Engers y Stern (2002) se observa que a medida que aumenta la edad del dependiente y del cuidador aumenta la probabilidad de recibir/proporcionar cuidados, pero cuanto mayor es la diferencia de edad entre ambos menor es la probabilidad de proporcionar cuidados de forma voluntaria.

Se ha considerado conveniente introducir los ingresos mensuales del dependiente como restricción de identificación en la ecuación de necesidades no satisfechas por dos motivos. En primer lugar, porque antes de la implantación del Sistema de Autonomía y Atención a la Dependencia, la recepción de servicios sociales públicos estaba siempre condicionada a la situación económica de la persona mayor (Casado et al., 2010). En segundo lugar, porque hay evidencia de otros artículos que apoyan la inclusión de esta variable. En LaPlante et al, (2008) se clasifica a los individuos en función de la proporción que guardan sus ingresos respecto del valor del Suplemental Security Income (SSI) y se observa que el hecho de tener un ingreso mensual por debajo del 300\% de la cuantía del SSI incrementa el número de horas de cuidados formales recibidos. Y para el caso de España, el trabajo de Otero et al. (2003), que analiza las necesidades no satisfechas de atención a domicilio entre la población mayor residente en Madrid, constata a través de una regresión logística que las necesidades no satisfechas están fuertemente correlacionadas con el hecho de percibir bajos ingresos.

A continuación se explica la construcción de cada una de las variables dependientes. 


\section{Problemas laborales}

En relación a la definición de los problemas laborales del cuidador, una de las preguntas de la Encuesta de Apoyo Informal es la siguiente: " $E l$ hecho de tener que responsabilizarse del cuidado de una persona mayor puede tener cierta incidencia en el desarrollo de la vida normal de la persona. En esta tarjeta hay una serie de consecuencias que pueden tener este tipo de situaciones. Me gustaría que me indicara aquéllas que a Vd, le ocurren por tener que ayudar a la persona de la que cuida: (i) no puede plantearse trabajar fuera de casa; (ii) ha tenido que dejar de trabajar; (iii) ha tenido que reducir su jornada de trabajo; (iv) su vida laboral se ha resentido (promoción, etc,); (v) tiene problemas para cumplir sus horarios". Denotamos por $P L_{i}$ una variable ordinal que mide "los problemas laborales del cuidador informal" y que puede tomar tres valores: (1) está ocupado y no tiene problemas laborales; (2) está ocupado, pero tiene problemas para cumplir horarios, su carrera laboral se ha resentido o ha tenido que reducir su jornada de trabajo; (3) ha dejado de trabajar, no puede plantearse trabajar fuera de casa.

Sin embargo, esta variable sólo se observa para aquellos individuos que estaban ocupados en el momento de convertirse en cuidadores informales. Por tanto, sólo observamos $P L_{i}$ si se cumple una determinada regla de selección. Esta regla de selección viene dada por una variable binaria $W_{i}$ que toma el valor 1 si el individuo estaba ocupado en el momento de convertirse en cuidador $\left(W_{i}=1\right)$, y el valor cero en caso contrario $\left(W_{i}=0\right)$. La variable de selección se genera de acuerdo con el siguiente modelo de variable latente:

$$
\begin{aligned}
& W_{i}^{*}=\Lambda_{i}^{\prime} \alpha+\omega_{i} \\
& W_{i}=\left\{\begin{array}{l}
1 \text { si } W_{i}^{*}>0 \\
0 \text { si } W_{i}^{*} \leq 0
\end{array}\right.
\end{aligned}
$$

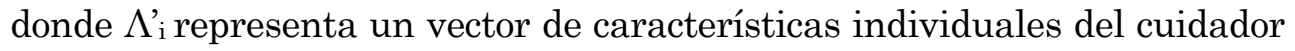
(sexo, edad, estudios, estado civil, situación profesional del sustentador principal (cuando es otra persona distinta del cuidador), hijos en el hogar, tamaño del municipio de residencia), $\alpha$ es un vector de coeficientes y $\omega_{i}$ es un término de error aleatorio. En consecuencia, la variable $P L_{i}$ no se observa cuando $W_{i}=0$ y toma un número limitado de valores, $P L_{i j}, j=1,2,3$ si $W_{i}=1$. Estas categorías están ordenadas como sigue: $P L_{i 1}>P L_{i 2}>P L_{i 3}$, aunque la diferencia entre cada par no tiene necesariamente una interpretación cardinal. La variable observada $\left(P L_{i}\right)$ se determina a partir de la definición de ciertos umbrales: 


$$
P L_{i}=\left\{\begin{array}{l}
\cdot \text { si } W_{i}=0 \\
1 \text { si } K J_{i}^{*}>\lambda_{1} \text { y } W_{i}=1 \\
2 \text { si } \lambda_{1}>K J_{i}^{*}>\lambda_{2} \text { y } W_{i}=1 \\
3 \text { si } \lambda_{2}>K J_{i}^{*} \text { y } W_{i}=1
\end{array}\right.
$$

donde $\lambda_{1}$ y $\lambda_{2}$ son constantes que se estiman junto con los otros parámetros del modelo.

\section{Horas diarias de cuidado informal}

Para la medición de los cuidados informales se utiliza la siguiente pregunta: "Cada día que atiende a esa persona mayor, ¿cuánto tiempo dedica al cuidado de esa persona? (i) menos de una hora; (ii) de 1 a 2 horas; (iii) de 3 a 5 horas; (iv) de 5 a 8 horas; (v) más de 8 horas, en ese caso, ¿cuántas?". Se ha definido la variable "horas de cuidados informales" tomando como referencia el punto medio del intervalo para las cuatro primeras categorías, es decir, 0,5, 1,5, 5 y 6,5 horas ${ }^{10}$, y para la categoría de más de 8 horas se ha empleado el número de horas exacto reportado por el cuidador (hasta llegar a 24 horas/día). En las estimaciones se utiliza esta variable en logaritmos.

\section{Necesidades no satisfechas}

En la literatura relativa a cuidados de la salud, se ha definido el concepto de necesidad como "aquellos requerimientos que permiten a los individuos alcanzar, mantener o recuperar un nivel aceptable de independencia social y calidad de vida" (Department of Health Social Services Inspectorate, 1991). Una definición más práctica es la que considera la necesidad como "la capacidad para beneficiarse de los servicios sociales" (Stevens y Gabay, 1991). Respecto a las "necesidades no satisfechas", Isaacs y Neville (1976) las describieron en relación a las sufridas por personas mayores como "el resultado de cuidados insuficientes para satisfacer las necesidades básicas de alimento, calor o limpieza al nivel que se lo hubiesen proporcionado esas mismas personas si hubieran podido" o "cuando los cuidados han sido proporcionados a costa de un esfuerzo desmesurado de sus familiares".

\footnotetext{
10 De los 1.137 individuos de la muestra, hay 24 que dedican menos de 1 hora al día a prestar cuidados, 161 dedican de 1 a 2 horas, 270 de 3 a 5 horas, 192 de 5 a 8 horas y 490 que dedican más de 8 horas diarias de cuidados. Para estos últimos se conoce el número exacto de horas.
} 
En este trabajo nos vamos a concentrar en las necesidades no satisfechas de Atención a Domicilio y Centros de Día ${ }^{11}$, y se considera que existe una necesidad no satisfecha cuando se solicita una ayuda formal y no se recibe, o bien se recibe, pero no en la cantidad o calidad deseada. Para definir las necesidades no satisfechas derivadas de un rechazo en la solicitud del servicio se utilizan dos preguntas de la encuesta: (i) "En esta tarjeta figura una lista de servicios o ayudas que las instituciones pueden prestar a las personas mayores dependientes, ¿cuál o cuáles ha solicitado alguna vez para la persona mayor de la que venimos hablando?”, (ii) “iy cuáles está utilizando o ha utilizado?". Consideramos que existe una necesidad no satisfecha cuando se responde afirmativamente a la primera pregunta (Atención a Domicilio, Centro de Día) y negativamente a la segunda. Para las necesidades no satisfechas derivadas de un insuficiente número de horas de prestación del servicio o de una deficiente calidad, nos remitimos a las respuestas para las siguientes tres preguntas "Dígame, por favor, la valoración de la ayuda que recibe en relación con las necesidades que Vd., tiene y de la persona a la que cuida (excelente, buena, regular, mala): (i) formación de la persona que le presta la ayuda, (ii) número de horas que le presta la ayuda, (iii) trato y amabilidad de la persona que le presta la ayuda". Por tanto, se considera que existe una necesidad no satisfecha por motivo de calidad y/o cantidad cuando se responde "regular" o "mala" a alguna de las anteriores preguntas.

Se define la variable binaria "necesidades no satisfechas" (NNS $\left.S_{i}\right)$ que toma el valor uno si el cuidador ha solicitado un servicio (Atención a Domicilio, Centro de Día), pero no lo recibe, o bien lo recibe, pero no cubre sus expectativas.

\section{Estructura de correlaciones}

Dado que suponemos que el hecho de ser cuidador y tener problemas laborales puede estar afectado por características inobservables que influyan simultáneamente sobre la probabilidad de estar trabajando, el número de horas de cuidado informal y la existencia de necesidades no satisfechas, la correlación entre los términos de error es distinta de cero. Suponemos que la correlación entre $\mathcal{E}_{1 i}, \varepsilon_{2 i}, \mathcal{E}_{3 i}$ y $\omega_{i}$ está inducida por un

\footnotetext{
11 No se modeliza el proceso de concesión de ayudas para adquisición de sillas de ruedas o camas articuladas, ni tampoco los servicios de comida y lavandería a domicilio, que son muy minoritarios, puesto que lo que se pretende estudiar es el efecto de la ausencia de cuidadores profesionales que ayuden al dependiente, pero también al cuidador y le permitan conciliar diferentes aspectos de su vida. Y no consideramos la atención residencial porque la institucionalización de la persona dependiente queda fuera del ámbito de estudio de este trabajo.
} 
término de heterogeneidad inobservable $\left(\mu_{i}\right)$ que afecta a la probabilidad de estar ocupado antes de convertirse en cuidador, a la incidencia de problemas laborales y necesidades no satisfechas, y a la cantidad de cuidados informales prestados, de forma que:

$\varepsilon_{3 i}=\mu_{i}+\varsigma_{i}$

$\varepsilon_{2 i}=\theta_{1} \mu_{i}+\zeta_{i}$

$\varepsilon_{1 i}=\theta_{2} \mu_{i}+\xi_{i}$

$\omega_{i}=\theta_{3} \mu_{i}+v_{i}$

Donde $\theta_{1}, \theta_{2}, \theta_{3} \in \mathfrak{R}$ son parámetros que se estiman al mismo tiempo que el modelo y que permiten cualquier tipo de correlación entre $P L, H I, N N S$ y $W$. Los errores idiosincráticos $\left(\zeta_{i}, \zeta_{i}, \zeta_{i}\right.$ y $\left.v_{i}\right)$ se distribuyen según una normal estándar y son ortogonales con respecto al término de heterogeneidad inobservable $\left(\mu_{i}\right)$.

El modelo se puede estimar por máxima verosimilitud simulada (Train, 2003). Se utiliza el simulador de Geweke-Hajivassiliou-Keane (GHK), que alcanza una buena precisión siempre que el número extracciones (draws) sea mayor o igual que la raíz cuadrada del tamaño muesstral (Cappelari y Jenkins, 2003). Para $\mathrm{N}=1.137$ se han realizado 200 extracciones por observación en la secuencia de simulación.

\subsection{Modelo probit ordenado con sesgo de selección y una variable endógenas}

Se ha considerado apropiado añadir este apartado porque al estimar el modelo anterior no ha resultado significativo el coeficiente de correlación entre "problemas laborales" y "horas de cuidado informal" $(\rho \text { (PL-HI) })^{12}$, indicando que las variables inobservables que afectan al número de horas de cuidado informal están incorrelacionadas con los inobservables que afectan a la aparición de problemas laborales entre cuidadores. Por consiguiente, la variable "horas de cuidado informal" es exógena con respecto a la existencia de problemas laborales.

Para elaborar este modelo se ha tomado como referencia el trabajo de Miranda y Bratti (2009). Por una parte, comparte con dicho modelo los siguientes rasgos: (1) la variable de interés del modelo es de naturaleza ordinal (problemas laborales), (2) dicha variable sólo es observada de acuerdo con una regla de selección (estar ocupado en el momento de convertirse en cuidador) y (3) en la especificación de la ecuación para la variable de interés aparece una variable potencialmente endógena (la

\footnotetext{
12 Dichos resultados se comentarán más detenidamente en la sección 5.2.
} 
existencia de necesidades no satisfechas). Por otra, se diferencia en que la ecuación para la variable de selección es más sencilla que la postulada por Miranda y Bratti (2009), puesto que la variable potencialmente endógena no aparece como explicativa. En relación a otros trabajos anteriores, este modelo presenta la innovación de considerar simultáneamente los problemas de endogeneidad y sesgo de selección (Miranda y Rabe-Hesketh (2006) sólo consideraban uno u otro), y la estimación se realiza mediante máxima verosimilitud con información limitada (a diferencia de Terza et al., (2008) que planteaban una estimación en dos etapas).

Por consiguiente, el modelo a estimar debería ser replanteado como:

$$
\begin{aligned}
& P L=\alpha_{1} \Omega_{1}+\kappa N N S+v_{1} \\
& N N S=\alpha_{2} \Omega_{2}+v_{2}
\end{aligned}
$$

donde $\Omega_{1}$ es un vector de variables exógenas que incluye las mismas que $\Pi_{1}$ más las horas de cuidado informal, $\Omega_{2}$ incluye las mismas variables que $\Omega_{1}$ más ingresos mensuales del dependiente, diferencia de edad y de sexo entre el cuidador y el dependiente, relación de parentesco, tamaño del municipio de residencia, tasa de cobertura del Servicio de Atención a Domicilio y de Centros de Día y porcentaje del tiempo de Atención a Domicilio dedicado a cuidados personales. La variable de selección $\mathrm{W}$ y la ecuación correspondiente se mantienen iguales a (2), y en relación a la estructura de correlación se supone que:

$$
\begin{aligned}
& v_{2 i}=\mu_{i}+\widetilde{\varsigma}_{i} \\
& v_{1 i}=\pi_{1} \mu_{i}+\widetilde{\xi}_{i} \\
& \omega_{i}=\pi_{2} \mu_{i}+\widetilde{v}_{i}
\end{aligned}
$$

Donde $\Pi_{1}, \Pi_{2} \in \mathfrak{R}$ son parámetros que se estiman al mismo tiempo que el modelo y que permiten cualquier tipo de correlación entre $P L, N N S$ y $W$. Los errores idiosincráticos $\left(\tilde{\zeta}_{i}, \tilde{\xi}_{i}\right.$ y $\left.\tilde{v}_{i}\right)$ se distribuyen según una normal estándar y son ortogonales con respecto al término de heterogeneidad inobservable $\left(\mu_{i}\right)$. Para expresar la contribución del individuo $i$ a la función de verosimilitud definimos un conjunto de variables binarias $d_{i j}, j=1,2,3$ que toman el valor 1 si $P L_{i}=j$, y cero en caso contrario: 


$$
\begin{aligned}
& L_{i}=\left[\Phi\left(\Lambda_{i}^{\prime} \alpha+\pi_{2} \mu_{i}\right) \cdot L_{i}^{*}\right]^{W_{i}} \cdot\left[1-\Phi\left(\Lambda_{i}^{\prime} \alpha+\pi_{2} \mu_{i}\right)\right]^{1-W_{i}} \\
& L_{i}^{*}=\int_{-\infty}^{\infty} \sum_{j=1}^{3} d_{i j} \Phi_{j}^{*}\left[N N S_{1} \Phi\left(\alpha_{2} \Omega_{2}+\mu_{i}\right)+\left(1-N N S_{1}\right)\left[1-\Phi\left(\alpha_{2} \Omega_{2}+\mu_{i}\right)\right] \cdot \phi\left(\mu_{i}\right) d \mu_{i}\right. \\
& \Phi_{j}^{*}=\left\{\begin{array}{l}
1-\Phi\left(\alpha_{1} \Omega_{1}-\tau_{1}+\pi_{1} \mu_{i}\right) \text { if } j=1 \\
\Phi\left(\alpha_{1} \Omega_{1}-\tau_{1}+\pi_{1} \mu_{i}\right)-\Phi\left(\alpha_{1} \Omega_{1}-\tau_{2}+\theta_{2} \mu_{i}\right) \text { if } j=2 \\
\Phi\left(\alpha_{1} \Omega_{1}-\tau_{2}+\pi_{1} \mu_{i}\right) \text { if } j=3
\end{array}\right.
\end{aligned}
$$

donde $\Phi$ es la distribución normal estándar. De la estimación, se obtienen tres coeficientes de correlación:

$$
\rho_{N N S-W}=\frac{\pi_{2}}{\sqrt{2\left(1+\pi_{2}^{2}\right)}} ; \rho_{N N S-P L}=\frac{\pi_{1}}{\sqrt{2\left(1+\pi_{1}^{2}\right)}} ; \quad \rho_{W-P L}=\frac{\pi_{1} \pi_{2}}{\sqrt{\left(1+\pi_{1}^{2}\right)\left(1+\pi_{2}^{2}\right)}}
$$

con los cuales se contrasta la exogeneidad de NNS respecto a PL y W $\left(\hat{\rho}_{N N S-P L}, \hat{\rho}_{N N S-W}\right)$ y la aleatoriedad de la muestra seleccionada $\left(\hat{\rho}_{W-P L}\right)$.

\section{Datos}

La fuente de datos utilizada es la Encuesta de Apoyo Informal, llevada a cabo por el IMSERSO en el año 2004. Esta encuesta se realizó mediante entrevista personal en el hogar a los cuidadores informales de personas mayores de 60 años. El cuidador y la persona dependiente pueden ser corresidentes o no. A partir de una muestra de 25.100 hogares españoles ${ }^{13}$, el tamaño muestral final fue de 1.504 entrevistas. Por consiguiente, todos los individuos entrevistados ya han tomado la decisión, en un momento anterior, de convertirse en cuidadores informales. De hecho, una de las limitaciones del trabajo es que con esta encuesta no se dispone de información sobre individuos que no sean cuidadores o personas dependientes.

Primero, para concentrarnos en el periodo de vida laboral de los cuidadores, se han eliminado las observaciones menores de 25 años (31 observaciones) o mayores de 64 años (336 observaciones). Por consiguiente, quedan 1.137 observaciones (1.008 mujeres y 129 hombres), Se define la variable "problemas laborales" como una variable ordinal tal y como se ha especificado en la sección anterior, y también se define una variable binaria "ocupado antes de ser cuidador" que toma el valor uno si el cuidador está ocupado en el momento de realizarse la encuesta o lo estaba antes, pero ha dejado su trabajo, por sus obligaciones con la

\footnotetext{
${ }^{13}$ Ceuta, Melilla y La Rioja fueron excluidas de la muestra.
} 
persona dependiente. En el Cuadro 1 se muestra la distribución de la muestra.

\begin{tabular}{lccc}
$\begin{array}{l}\text { Cuadro 1. Distribución de la muestra } \\
\text { problemas laborales, }\end{array}$ & por & severidad de los & Cuidadores \\
& $\begin{array}{c}\text { Cuidadores } \\
\text { mujeres }\end{array}$ & $\begin{array}{c}\text { Total } \\
\text { cuidadores }\end{array}$ \\
\hline No ocupados antes de convertirse en cuidador & 26 & 256 & 282 \\
Ocupados antes de convertirse en cuidador & 103 & 752 & 855 \\
$1^{\circ}$ ) No problemas laborales & 14 & 131 & 180 \\
$2^{\circ}$ Probl. laborales (horarios, jornada, ascensos) & 74 & 320 & 394 \\
$3^{\circ}$ Se han visto obligados a dejar de trabajar & 15 & 301 & 281 \\
\hline Total & 129 & 1,008 & 1,137 \\
\hline
\end{tabular}

Fuente: Elaboración propia a partir de la Encuesta de Apoyo Informal (2004).

\section{Medición del nivel de dependencia}

En relación a cómo se ha medido el grado de dependencia, en este trabajo se ha aplicado el Baremo de Valoración mencionado por la Ley 39/2006, de 14 de diciembre, de Promoción de la Autonomía Personal y Atención a las Personas en Situación de Dependencia, que ha sido aprobado por el Real Decreto 504/2007 de 20 de abril.

Este Baremo de Valoración permite determinar el grado de dependencia (moderada, severa o grande) ${ }^{14}$ de acuerdo con el grado de autonomía a la hora de realizar actividades cotidianas, tomando en consideración, la necesidad de apoyo o supervisión, así como la existencia de limitaciones cognitivas (art. 26 y 27).

A la hora de comparar las tareas del Baremo de Valoración con las preguntas de la Encuesta de Apoyo Informal se tomado como referencia el trabajo de Vilaplana (2009) con la ventaja que supone poder clasificar a la población dependiente de acuerdo con la clasificación vigente, pero también con las limitaciones que impone el hecho de que la información disponible está basada en las respuestas de un cuidador informal y no de un evaluador oficial. El Cuadro 2 muestra la clasificación obtenida.

\footnotetext{
${ }^{14}$ Se considera que un individuo padece dependencia moderada cuando necesita ayuda para la ejecución de actividades de la vida diaria al menos una vez al día. Sufre dependencia severa cuando necesita ayuda dos o tres veces al día, y gran dependencia cuando necesita ayuda varias veces al día, y debido a una completa pérdida de la autonomía física, mental, intelectual o sensorial, requiere de apoyo permanente. Asimismo, se identifican dos niveles de dependencia dentro de cada grado. El primer nivel corresponde a aquellos individuos que pueden realizar la actividad sin el apoyo directo de una tercera persona, mientras que el segundo nivel se refiere a las situaciones en donde el dependiente precisa de apoyo específico.
} 
Cuadro 2. Baremo de Valoración para la determinación del nivel de dependencia

\begin{tabular}{ll|c|c}
\hline & & Ley de Dependencia & Encuesta de Apoyo Informal \\
\hline No Dependientes & & $0-24$ & $538(35,77 \%)$ \\
\hline Moderado & Nivel 1 & $25-39$ & $275(18,28 \%)$ \\
& Nivel 2 & $40-49$ & $172(11,44 \%)$ \\
\hline \multirow{2}{*}{ Severo } & Nivel 1 & $50-64$ & $242(16,09 \%)$ \\
& Nivel 2 & $65-74$ & $139(9,24 \%)$ \\
\hline \multirow{2}{*}{ Grande } & Nivel 1 & $75-89$ & $124(8,24 \%)$ \\
& Nivel 2 & $90-100$ & $14(0,94 \%)$ \\
\hline
\end{tabular}

Fuente: Elaboración propia a partir de

$\underline{\text { www,seg-social,es/imserso/dependencia/manualusoBVD,pdf }}$

Para corroborar la fiabilidad de estos resultados se ha realizado una comparación con las proyecciones para el año 2005 elaboradas por el INE a partir de la Encuesta de Discapacidades, Deficiencias y Estado de Salud (1999).

Cuadro 3. Comparación de la estimación de los grados de dependencia con las proyecciones elaboradas a partir del Libro Blanco de la Dependencia

$\begin{array}{cc}\text { Proyecciones para } 2005 \text { a partir de la Encuesta de } & \text { Encuesta de Apoyo } \\ \text { Discapacidades, Deficiencias y Estado de Salud } & \text { Informal (2004) }\end{array}$ (1999)

\begin{tabular}{|c|c|c|c|c|}
\hline & $65 \mathrm{y}+$ años ${ }^{(a)}$ & $\%$ & $65 \mathrm{y}+$ años & $\%$ \\
\hline Grande & 163,334 & $15,39 \%$ & 137 & $9,45 \%$ \\
\hline Severa & 292,105 & $27,52 \%$ & 365 & $25,19 \%$ \\
\hline Moderada & 371,112 & $34,96 \%$ & 438 & $30,23 \%$ \\
\hline Total & $1,061,404^{(b)}$ & & $1,449^{(\mathrm{c})}$ & \\
\hline
\end{tabular}

(a): Grados de dependencia de la página 89 del "Atención a las Personas en Situación de Dependencia" (2004).

(b): Corresponde al número de personas con discapacidades básicas o instrumentales para actividades de la vida diaria (página 89; "Atención a las personas en situación de Dependencia, Libro Blanco" (2004)).

(c): Para la construcción de esta Tabla 3 se ha restringido la muestra a los mayores de 65 años.

Se observa que existe un alto nivel de consistencia para la discapacidad moderada y severa $(34,96 \%$ y $27,52 \%$, respectivamente, de acuerdo con las proyecciones del INE). La disparidad que se aprecia en la gran dependencia $(15,39 \% \mathrm{vs}, 9,45 \%)$ puede deberse a que las proyecciones incluyen a las personas mayores institucionalizadas mientras que la Encuesta de Apoyo Informal sólo considera a los mayores residentes en el hogar. 


\section{Estadísticos descriptivos}

En la Tabla 1 del Apéndice se observa que atendiendo al género y edad de la persona que recibe cuidados, para todos los casos considerados, aproximadamente el $70 \%$ de las personas que reciben cuidados son mujeres con una edad media de 80 años. En cambio sí existen diferencias significativas en el estado de salud, puesto que los cuidadores ocupados con problemas o que han dejado de trabajar cuidan de personas dependientes que sufren en mayor medida las siguientes discapacidades: no puede comer solo $(31,94 \%, 32,90 \%)$, no puede utilizar el servicio $(23,38 \%, 27,73 \%)$, no le importa la limpieza personal $(25,88 \%, 27,12 \%)$, no puede asearse por sí solo (70,93\%, 73,39\%), no puede vestirse/desvestirse $(46,19 \%, 56,95 \%)$, no puede levantarse o acostarse de la cama $(46,19 \%$, $56,95 \%)$, no puede preparar comidas $(34,83 \%, 37,13 \%)$. También se detecta una mayor presencia de enfermos mentales $(40,76 \%, 35,90 \%)$ o que pueden ser clasificados como dependientes severos de nivel $2(12,48 \%$, $11,25 \%)$ o grandes dependientes de nivel 1 (8,60\%, 14,65\%),

Respecto a los cuidadores que han dejado de trabajar, el 95,97\% son mujeres, presentan el mayor porcentaje de "sin estudios" $(17,59 \%)$, no suelen ser sustentadores del hogar (10,08\%), suelen llevar más años de cuidado (el 13,72\% lleva más de 12 años) y dedican más horas al día (12,40 horas). Por el contrario el $42,43 \%$ de los ocupados sin problemas lleva menos de 2 años de cuidados, es sustentador del hogar $(48,31 \%)$ y vive en capitales de provincia $(40,71 \%)$.

\section{Resultados}

\subsection{Test de diagnóstico}

Para contrastar la validez y robustez de los instrumentos se han llevado a cabo cinco test (Tabla 3 del Apéndice). En primer lugar se han calculado estos estadísticos para HI y NNS cuando se estima el modelo con dos variables endógenas. Más adelante, y debido a la no significatividad de la correlación $\rho(\mathrm{PL}-\mathrm{HI})$ se estima el modelo con un sola variable endógena (NNS) y se vuelven a computar los mismos estadísticos. En todos los casos se llega a la misma conclusión: los instrumentos son relevantes (test $\mathrm{F}$ de los instrumentos excluidos) y están fuertemente correlacionados con la variable endógena ( $R^{2}$ parcial). Además, el test de J-Hansen nunca es significativo por lo que suponemos que el modelo está correctamente especificado, no se acepta 
la hipótesis nula del test de Kleibergen-Paap (Wald y LM) por lo que no se puede aceptar que el modelo esté infraidentificado, y finalmente, no se acepta en ningún caso la no significatividad conjunta de los regresores endógenos (test de Anderson-Rubin).

\subsection{Resultados y predicciones}

La Tabla 2 del Apéndice muestra los resultados de la estimación del modelo propuesto. En relación a los términos de correlación, las variables inobservables que afectan a la aparición de problemas laborales están negativamente correlacionadas con los inobservables que afectan al hecho de estar ocupado antes de ser cuidador y positivamente correlacionadas con los inobservables que influyen en la probabilidad de sufrir necesidades no satisfechas. Las horas de cuidado informal sólo ejercen un efecto causal sobre los problemas laborales ya que el coeficiente de correlación entre ambas variables no es significativo, y por consiguiente, no puede considerarse endógena. A tenor de estos resultados y los de la sección anterior, se ha vuelto a estimar el modelo considerando que sólo existe una variable endógena (NNS). El signo de la correlación W-PL confirma la existencia de sesgo de selección, así como la endogeneidad de las necesidades no satisfechas respecto a los problemas laborales y al estado de ocupación. Este último resultado apoyaría la tesis de que los cuidadores que se encuentran ocupados son más propensos a solicitar ayudas formales con respecto a los que no estaban trabajando antes de convertirse en cuidador, o más proclives a considerar que los servicios formales recibidos no les ayudan lo suficiente a compatibilizar trabajo y cuidados.

En la Tabla 4 del Apéndice se muestran las probabilidades marginales de acuerdo con las estimaciones del probit ordenado con una variable de endógena y sesgo de selección. La probabilidad de no tener problemas laborales o tener algunos problemas es mayor para los hombres, y lo contrario sucede para la probabilidad de dejar de trabajar, que es superior para las mujeres. Además, para los hombres la probabilidad de no tener problemas laborales es un $11,50 \%(22,79 \%)$ superior a la probabilidad de tener problemas laborales (dejar de trabajar), mientras que para las mujeres disminuye un $39,78 \%$ $(67,70 \%)^{15}$.

Si relacionamos el sexo del dependiente y del cuidador, se observa que para las mujeres las probabilidades estimadas no varían de

\footnotetext{
${ }^{15}$ Lai y Leonelko (2007) también hallaron que las mujeres soportan mayores perjuicios
} laborales que los hombres. 
manera significativa, tanto si la persona dependiente es hombre o mujer, pero en el caso de los hombres, la probabilidad de no tener problemas laborales disminuye un 7,97\% y la probabilidad de dejar de trabajar aumenta un $16,69 \%$ cuando la persona a la que cuida es una mujer. Este resultado coincide con el de Cordingley y Web (1997) de que en ocasiones la diferencia de género puede generar cierta incomodidad a la hora de comentar las necesidades de cuidado o recibir atención de otra persona de distinto sexo.

En relación a la edad, cuanto más joven es el cuidador, mayor es la probabilidad de no tener problemas laborales y menor es la probabilidad de dejar de trabajar, de manera que para cuidadores de entre 50 y 64 años la probabilidad de no tener problemas respecto a sí tenerlos (dejar el trabajo) disminuye un 46,02\% (74,41\%). Si calculamos la diferencia entre la edad de la persona dependiente y el cuidador, la probabilidad de que no haya problemas laborales es máxima cuando hay una diferencia de 50 años de edad. Por el contrario, la probabilidad de haberse visto obligado a dejar el trabajo es máxima cuando hay una diferencia de menos de 10 años de edad ${ }^{16}$. Las mayores diferencias de la probabilidad de no tener problemas respecto a las otras dos categorías también ocurren cuando la diferencia es inferior a 10 años.

Atendiendo a la relación de parentesco, el esposo/hijo/yerno siempre tiene mayor probabilidad no tener ningún problema laboral o tener algunos, y menor probabilidad de haber dejado el trabajo respecto a la esposa/hija/nuera. En términos absolutos, la esposa tiene una probabilidad considerablemente inferior a la estimada para la hija/nuera, pero la tasa de variación dentro de cada pareja (esposoesposa, hijo-hija, yerno-nuera) revela que existe mayor diferencia entre esposo-esposa, a continuación entre yerno-nuera, y finalmente entre hijo-hija ${ }^{17}$.

Los cuidadores que trabajan como profesionales independientes presentan la mayor probabilidad de no tener problemas $(63,92 \%$ superior a la de los trabajadores no cualificados y $91,35 \%$ superior a la de los empresarios sin asalariados). Las categorías de "empresarios sin asalariados" y "trabajadores no cualificados" se revelan como las más vulnerables al efecto de los problemas laborales, puesto que la probabilidad de no tener problemas respecto a sí tenerlos disminuye un

\footnotetext{
${ }^{16}$ El rango de la variable "diferencia de edad" calculado como la edad del dependiente menos edad del cuidador va de -3 años a 76 años.

17 Por ejemplo, para el suceso "no tener problemas laborales" la probabilidad del esposo es 523\% superior a la de la esposa, la del yerno 158\% superior a la de la nuera y la del hijo $106 \%$ superior a la de la hija.
} 
$33,53 \%$ y $20,46 \%$, respectivamente, y la probabilidad de no tener problemas respecto a haber dejado el trabajo disminuye un 34,96\% y $17,04 \%$, respectivamente.

Los cuidadores de personas dependientes con patologías cardiovasculares, respiratorias o cáncer obtienen una probabilidad de no tener problemas laborales cercana al $20 \%$ frente a un $10 \%$ para los cuidadores de enfermos mentales. Aunque las probabilidades de tener algún tipo de problemas de conciliación son aproximadamente las mismas para todas las patologías consideradas, donde sí se aprecian diferencias es en la disminución de la probabilidad de no tener problemas respecto a dejar de trabajar (un $80 \%$ para enfermos mentales y un $60 \%$ en el resto de las enfermedades).

$\mathrm{Si}$ relacionamos los problemas laborales con el grado de dependencia, se aprecia que la probabilidad de que no se sufran problemas laborales disminuye un $78,97 \%$ (83,54\%) al pasar de "no dependiente" a "gran dependiente de nivel 1 (nivel 2)". La probabilidad de que existan algunos problemas aumenta un $52,86 \%$ al pasar de "no dependiente" a "severo de nivel 2" y disminuye un $27,06 \%$ al comparar "severo de nivel 2" con "gran dependiente de nivel 2". Esta reducción se debe a que la probabilidad de tener problemas respecto a la probabilidad de dejar el empleo disminuye un 30\% para los "severos de nivel 2" y un $60 \%$ para todos los grandes dependientes.

Los resultados relativos a la carga de cuidados (horas diarias y años de cuidados) revelan que a medida que aumenta cualquiera de estas dos variables, disminuye la probabilidad de no tener problemas laborales y aumenta la probabilidad de sufrirlos o de haber dejado de trabajar. Por ejemplo, al pasar de "menos de 1 hora/día" a "más de 8 horas/día" (o al comparar "menos de 2 años de cuidados" con "más de 12 años de cuidados") la probabilidad de no tener problemas laborales se reduce en un 52,85\% (-37,67\%). Por el contrario, para estas mismas categorías la probabilidad de haber dejado de trabajar aumenta en un $49,20 \%$ y $24,10 \%$, respectivamente. Comparando estos resultados con los de Casado et al, (2010), ellos observaban que la probabilidad de trabajar a tiempo completo no disminuía de forma significativa durante el primer año, sino más bien en años sucesivos. En nuestro caso, la probabilidad de dejar de trabajar durante los primeros dos años alcanza el 48,34\%, y aunque luego asciende hasta el 59,99\% para cuidadores que llevan más de 12 años de cuidado, no podemos saber en qué momento dejaron de trabajar. Por consiguiente, habría que considerar que desde el estudio de Casado et al, (2010) que abarca el periodo 19942001, hasta el año 2004 se ha producido un empeoramiento de las 
posibilidades de conciliación de trabajo y cuidados para las cuidadoras españolas.

La categoría "de 3 a 5 horas diarias de cuidados" marca una fuerte caída en la probabilidad de no tener problemas laborales (de $30,42 \%$ a $22,70 \%$ ) y un incremento significativo en la probabilidad de dejar de trabajar (de 35,63\% a 45,83\%). Dado que la probabilidad de tener problemas laborales engloba la circunstancia de reducción de la jornada laboral, estos resultados concuerdan con los de Casado et al. (2010) según los cuales la incidencia de la jornada parcial no es progresiva conforme se incrementa el número de horas de cuidado, sino que más bien estaríamos ante un proceso de transición entre la situación de estar trabajando y el estado de inactividad o desempleo.

Por último, el trabajo de Casado et al. (2010) analiza los efectos sobre la participación laboral en función del número de horas semanales de cuidado (menos de 14 , de 14 a 28 y más de 28 ) y concluye que a partir de 28 horas semanales la probabilidad de no trabajar aumenta 4,5 puntos porcentuales, mientras que la probabilidad de trabajar a jornada completa (parcial) se reduce en $20,5(17,5)$ puntos porcentuales. En nuestro caso, se han relacionado horas y años de trabajo simultáneamente con las probabilidades de no tener problemas laborales, tener dificultades para continuar trabajando y haber dejado de trabajar (Gráficos 1, 2 y 3 del Apéndice), A la vista de los gráficos pueden distinguirse dos grupos de cuidadores. Los que llevan "hasta 12 años de cuidado" (categorías "menos de 2 años", "de 2 a 4 años" y "de 4 a 12 años") para los que se observa que, condicionado a proporcionar entre 3 y 5 horas diarias de cuidado, la probabilidad de dejar de trabajar aumenta considerablemente y se sitúa entre 0,41\% y $0,49 \%$, mientras que la probabilidad de no tener problemas laborales experimenta un fuerte descenso y toma valores entre $0,19 \%$ y $0,7 \%$ (lo cual estaría en la línea de las 28 horas semanales de Casado et al. (2010)). Para los cuidadores que llevan más de 12 años de cuidado la probabilidad de no estar trabajando (o de no tener problemas laborales) es marcadamente superior (inferior) para cargas de horas de cuidado menores a 3-5 horas al día. Estas evidencias indicarían que para los cuidadores españoles de personas mayores no parece cumplirse la teoría de la "carrera del cuidador" ya que no se observa una progresiva pauta de acomodamiento a la carga de cuidados (Aneshensel et al,, 1993).

La incidencia de los problemas laborales no es igual, tanto en lo que se refiere a la comunidad autónoma, como al tamaño del municipio de residencia. Atendiendo al primer aspecto, los valores máximos y mínimos de las probabilidades predichas siempre corresponden a Madrid y Navarra. En términos relativos, la probabilidad de no tener 
problemas en comparación con sí tenerlos disminuye un $46,02 \%$ en Castilla La Mancha y un 45,24\% en Extremadura; la probabilidad de no tener problemas respecto a dejar el empleo disminuye un $77,48 \%$ en Murcia y un 75,93\% en Castilla La Mancha; y la probabilidad de tener problemas laborales respecto a dejar el trabajo disminuye un $62,32 \%$ en Murcia y un 55,42\% en Castilla La Mancha.

El tamaño del municipio de residencia juega un papel relevante para los tamaños extremos: aumenta un $68,15 \%$ la probabilidad de no tener problemas y reduce un 18,33\% la probabilidad de dejar el empleo, al pasar de municipios de menos de 2.000 habitantes a capitales de provincia. Para la probabilidad de tener problemas laborales no se aprecian diferencias significativas en términos absolutos (en todos los casos, la probabilidad se encuentra en torno a 0,30), pero sí cuando se comparan con las otras dos. Por ejemplo: la probabilidad de no tener problemas es un $52,28 \%$ más pequeña que la probabilidad de sí padecerlos en los municipios de menos de 2.000 habitantes, pero sólo un $21,47 \%$ menor en las capitales de provincia.

En relación a las necesidades no satisfechas, la probabilidad de no tener problemas es sólo de 0,0390 $(0,0528)$ cuando hay una NNS de Atención a Domicilio (Centro de Día), frente a una probabilidad para haberse visto obligado a dejar el trabajo de 0,7863 (0,7642). Llama la atención que exista escasa diferencia entre la NNS cuando no se recibe la Atención a Domicilio o cuando se recibe, pero no se está satisfecho con la calidad, puesto que las probabilidades estimadas en este caso son 0,0398 y 0,0621 , respectivamente, para no tener problemas, y 0,7863 y 0,7338 para dejar el trabajo. Entre las teorías de que explican la relación entre cuidados formales e informales, la teoría de la sustitución sostiene que los cuidados informales disminuyen a medida que se reciben cuidados formales (Greene, 1983). En este caso, se trataría de la situación opuesta: el cuidador informal tiende a salir del mercado de trabajo para prestar cuidados y suplir la carencia de cuidados formales cuando estos han sido solicitados o se reciben en cantidad o calidad suficiente. Por otra parte, el porcentaje del tiempo de Atención a Domicilio dedicado a cuidados personales ${ }^{18}$ revela que la probabilidad de no tener problemas laborales aumenta un $67,99 \%$, y la probabilidad

\footnotetext{
18 En la Encuesta de Apoyo Informal se sabe si la persona dependiente recibe Atención a Domicilio y si el cuidador está satisfecho con la misma, pero no se le pregunta cómo reparte el cuidador formal su tiempo entre cuidados personales y tareas domésticas. No obstante, en "Las personas mayores en España" (2004) existe información por comunidades autónomas sobre el reparto del tiempo de la Atención Domiciliaria. El porcentaje del tiempo dedicado a cuidados personales oscila entre 20\% para Extremadura y $80 \%$ para Navarra.
} 
de dejar de trabajar disminuye un 19,40\% cuando la fracción del tiempo destinada a cuidados personales pasa de menos del $25 \%$ a más del $75 \%$.

El hecho de que la probabilidad de estar ocupado sin problemas cuando hay necesidades no satisfechas alcance unos valores tan bajos nos lleva a preguntarnos cuál sería el impacto sobre la situación laboral de los cuidadores si se solucionaran sus problemas de necesidades no satisfechas. Para ello se comparan dos situaciones. Las tres primeras columnas de la Tabla 5 del Apéndice muestran las probabilidades por comunidades autónomas para el subconjunto de cuidadores que han declarado padecer necesidades no satisfechas ${ }^{19}$. A continuación se ha computado cuál sería la probabilidad de padecer problemas laborales en el caso de que se solucionaran dichas necesidades no satisfechas ${ }^{20}$.

Al comparar las tres primeras columnas de la Tabla 5 con las probabilidades por comunidades de la Tabla 4, se observa que son mayores las obtenidas para el conjunto de la muestra en el caso de no tener problemas laborales o tener algunos, mientras que son mayores las de la Tabla 5 para haber dejado de trabajar. Centrándonos en la Tabla 5 y comparando las situaciones con necesidades no satisfechas y una vez que éstas se han solucionado se aprecia un considerable incremento de la probabilidad de no tener problemas laborales (entre $295,95 \%$ para Baleares y $1625,67 \%$ para Navarra) y de la probabilidad de tener algunos problemas (entre 20,77\% para Baleares y $301,88 \%$ para Navarra) y una disminución de la probabilidad de dejar de trabajar (entre $-47,25 \%$ para Navarra y $-60,20 \%$ para Extremadura).

\section{Conclusiones}

El presente trabajo ha analizado el grado en el que las tareas que conllevan los cuidados informales condicionan tanto la continuidad en el puesto de trabajo como la posibilidad de ejercer una actividad laboral sin restricciones. Para ello se ha utilizado la Encuesta de Apoyo Informal (2004) que proporciona valiosa información desde el punto de

19 Hay 298 individuos que estaban ocupados antes de ser cuidadores y que tienen necesidades no satisfechas: 40 sin problemas laborales, 125 con problemas laborales y 133 que han dejado de trabajar.

20 (1) Que aquellos a los que se les denegó el servicio de Atención a Domicilio o de Centro de Día, recibieran la prestación solicitada, y (2) Que aquellos que recibían Atención Domiciliaria, pero consideraban que el trato recibido no era adecuado, o que la cualificación del cuidador formal o el número de horas eran insuficientes, estuvieran satisfechos con el servicio recibido. Las columnas 4, 5 y 6 muestran las probabilidades predichas en este escenario exclusivamente para el subconjunto de cuidadores que partían de una situación con necesidades no satisfechas. 
vista del dependiente, ya que permite clasificar su grado de dependencia de acuerdo con el Baremo de Valoración aprobado a partir de la implantación del Sistema de Autonomía y Atención a la Dependencia (SAAD), como desde el punto de vista del cuidador, puesto que se conoce su situación profesional, número de horas y años de cuidado. También se han incorporado variables referentes a la cobertura de los servicios sociales para personas dependientes (Atención a Domicilio, Centros de Día) con el propósito de comprobar cómo las diferencias regionales y la aparición de necesidades no satisfechas de ayuda formal pueden tener un impacto en la calidad de vida de las personas dependientes y en las facilidades para conciliar vida laboral y de cuidados.

A la hora de determinar un umbral a partir del cuál surgen problemas de conciliación entre cuidados informales y vida laboral, los resultados de este trabajo concuerdan con los de otros autores. Carmichael y Charles (2003) constataron que una carga de cuidados de menos de 10 horas/semana no tenía un impacto significativo sobre la oferta de trabajo, mientras que los trabajos de Joshi (1995) y Casado et al. (2010) evidenciaron que a partir de 20 ó 28 horas a la semana los cuidadores encontraban crecientes dificultades para continuar con su trabajo. En este sentido, Arksey et al, (2005) confirmaron que para una intensidad de cuidados de entre 20 y 49 horas/semana el porcentaje de cuidadores que permanecían ocupados disminuía a la mitad. Por otra parte, el hecho de que los problemas laborales tiendan a manifestarse, tanto si se lleva cuidando menos de 2 años, como más de 12 a partir de 3-5 horas diarias de cuidado coincide con los resultados de Casado et al. (2010) de que a partir de un año de cuidados ya se observan efectos sobre la participación laboral.

La preocupación por evitar la salida temporal o definitiva del mercado de trabajo es compartida por numerosos países (Alemania, Austria, Países Bajos, Reino Unido, Suecia) que han introducido reformas facilitando la reducción del número de horas de trabajo o la concesión de permisos, garantizando siempre la recuperación de las condiciones normales de trabajo una vez finalizado el periodo de cuidados (CESEP, 2004). En España, la igualdad efectiva entre hombres y mujeres cuidadores parece bastante lejana, ya que a pesar de que la Ley Orgánica 3/2007, de 22 de marzo aprobó el derecho de una reducción de jornada entre un octavo y un $50 \%$ en el trabajo, la probabilidad de dejar el trabajo es un $75 \%$ superior para las mujeres que para los hombres. Por otra parte, los empresarios sin asalariados y los trabajadores no cualificados son una de las categorías más 
vulnerables a sufrir problemas y en estos casos la reducción de horas puede suponer también un importante menoscabo económico.

Para intentar paliar estos problemas, en muchos sistemas de cuidados de largo plazo, se ha creado una prestación económica para el cuidador que incluye cotizaciones a las Seguridad Social para garantizar una futura pensión de jubilación, o se permite la posibilidad de elegir entre prestaciones monetarias y en especie ${ }^{21}$, con el fin de que la persona dependiente remunerase los servicios de su cuidador familiar, o de que se recibieran cuidados formales que permitieran disminuir las necesidades no satisfechas y la continuidad del cuidador en el mercado laboral.

El hecho de que el SAAD haya determinado los intervalos horarios de Atención a Domicilio correspondientes a cada grado y nivel de dependencia, y que para cada beneficiario se diseñe un Programa Individual de Atención acorde con sus necesidades y preferencias, debería suponer, en principio una reducción de las necesidades no satisfechas y de su repercusión sobre los cuidadores informales. Sin embargo, los últimos datos del Sistema de Información del SAAD revelan que de las 742.974 prestaciones reconocidas (octubre 2010), el 49,19\% (72,84\% en Murcia) correspondían a la prestación para cuidados en el entorno familiar. Estos datos plantean una cuestión en la que sería muy interesante profundizar cuando se dispusiera de datos posteriores a la implantación del SAAD (enero 2007), y es si este reconocimiento en términos económicos al trabajo desempeñado por los cuidadores pudiera estar generando un efecto encubierto en la forma de salida del mercado de trabajo o relegación de los compromisos laborales. En este contexto el bienestar futuro del cuidador pudiera quedar gravemente constreñido puesto que la duración de los cuidados informales es altamente imprecisa.

\section{Bibliografía}

Anderson, T, y Rubin, H, (1949): "Estimation of the parameters of a single equation in a complete system of stochastic equations", Annals of Mathematical Statistics, 20, pp. 46-63.

${ }^{21}$ Véanse tablas comparativas sobre sistemas de protección social en Employment Social Affairs and Equal Opportunities. Social Protection and Social Inclusion (European Commission):

http://ec.europa.eu/employment social/missoc/db/public/compareTables.do?lang=en 
Anheshensel. C., Pearlin, L. y Schufer, R. (1993): "Stress, role captivity and the cessation of caregiving", Health and Social Behaviour, 34, pp. 5470 .

Arber, S, y Ginn, J, (1995): "Gender differences in informal caring", Health and Social Care in the Community, 3, pp. 19-31.

Arksey, H,. Kemp, P,. Glendinning, C,. Kotchetkova, I, y Tozer, R,. (2005): "Carers' aspirations and decisions around work and retirement", Department of Work and Pensions, Research Report No, 290, Corporate Document Services.

Baldwin, S, (1985): The cost of caring, Routlegde \& Kegan Paul, London.

Blundell, R, y Smith, R, (1994): "Coherency and estimation in simultaneous models with censored or qualitative dependent variables", Journal of Econometrics, 64, pp. 355-373.

Boaz, R, y Muller, C, (1992): "Paid work and unpaid help by caregivers of the disabled and frail elders", Medical Care, 30, pp. 149-158.

Cappelari, L, y Jenkins, S, (2003): "Multivariate probit regression using simulated maximum likelihood", The Stata Journal 3(3).

Carmichael, F, y Charles, S, (1998): "The labor market costs and community care", Journal of Health Economics, 17, pp. 747-765.

Carmichael, F, y Charles, S, (2003): "Benefit payments, informal care and female labour supply”, Applied Economic Letters, 10, pp. 411-415.

Casado, D,. García-Gómez, P, y López, A, (2010): "Labour and income effects of caregiving across Europe: an evaluation using matching techniques", SERIEs: Journal of the Spanish Economic Association, DOI: 10,1007/s13209-009-0008-5.

Centre for European Social and Economic Policy (CESEP), (2007): "Exploring the synergy between promoting active participation in work and in society and social, health and long-term care strategies", Contract Reference 2006/030 (2006/S 123-130484)

Cordingley, L, y Webb, C, (1997): "Independence and ageing", Reviews in Clinical Gerontology, 7, pp. 137-146.

Crespo, L, (2008): "Caring for parents and employment status of European mid-life women”, CEMFI Working Paper 0615.

Department of Health Social Services Inspectorate, (1991): Care management and assessment: practitioners' guide, HMSO, London. 
Doty, P,. Jackson, M, y Crown, W, (1998): "The impact of female caregivers' employment status on patterns of formal and informal eldercare", The Gerontologist, 38, pp. 331-341.

Dweyer, J, y Coward, R, (1991): "A multivariate comparison of the involvement of adult sons versus daughters in the care of impaired parents", Journals of Gerontology, Social Sciences, 46, pp. S259-S269.

Engers, M, y Stern, S, (2002): "Long-term care and family bargaining", International Economic Review, 43(1), pp. 73-114.

Ettner, S, (1996): "The opportunity costs of elder care", The Journal of Human Resources, 31, pp. 189-205.

Gallagher, S, y Mechanic, D, (1996): "Living with the mentally ill: effects on the health and functioning of other household members", Social Science and Medicine, 42, pp.1691-1701.

Greene, V, (1983): "Substitution between formally and informally provided care for the impaired elderly in the community", Medical Care, 21, pp. 609-619.

Hansen L, (1982): "Large sample properties of generalized method of moments estimators", Econometrica, 50(3), pp. 1029-1054.

Heckman, J, (1978): "Dummy endogenous variables in a simultaneous equation system”, Econometrica, 46, pp. 931-959.

Heitmueller, A, y Michaud, P, (2006): "Informal care and employment in England: evidence from the British Household Panel Survey", IZA DP, No, 2010.

Horowitz, A, (1985): "Sons and daughters as care-givers to older parents: differences in role performance and consequences", The Gerontologist, 25, pp. 612-617.

Isaacs, B, y Neville, Y, (1976): "The measurement of need in older people", Scottish Health Service Studies, No, 34, Edinburgh: Scottish Home and Health Department.

Joshi, H, (1995): "The labour market of unpaid caring: conflict and compromise", En I, Allend y L, Perkins (comps,), The future of family care for older people, HMSO, London

Kleibergen F, y Paap, R, (2006): "Generalized reduced rank tests using the singular value decomposition", Journal of Econometrics, 133, pp. 97-126. 
Kolodinski, J, y Shirey, L, (2000): "The impact of living with an elder parent on adult daughter's labor supply and hours of work", Journal of Family and Economic Issues, 21, pp. 149-175.

Lai, W, y Leonelko, W, (2007): "Effects of caregiving on employment and economic costs of Chinese family caregivers in Canada", Journal of Family and Economic Issues, 28, pp. 411-427.

LaPlante, M, P,. Kaye, H, S,. Kang, T, y Harrington, C, (2004): "Unmet need for personal assistance services: estimating the shortfall in hours of help and adverse consequences”, Journal of Gerontology: Social Sciences, 59B(2), pp. S98-S108.

Maddala, G, (1983): Limited dependent and qualitative variables in Econometrics, Cambridge University Press, Cambridge.

McGarry, K, (2003): "Does caregiving affect work? Evidence based on prior labor force experience", JCER-NBER Conference.

Meyerhoefer, C. D., Pylypchuck, Y. (2008): "Does participation in the Food Stamp Programa increase the prevalence of obesity and health care spending?. American Journal of Agricultural Economics, 90(2), 287-305.

Miranda, A, y Bratti, M, (2009): "Selection endogenous dummy ordered probit and selection endogenous dummy dynamic ordered probit models", Stata Users' Group, United Kingdom Stata Users' Group Meeting 2009, No. 1.

Miranda, A, y Rabe-Hesketh, S, (2006): "Maximum likelihood estimation of endogenous switching and sample selection models for binary, ordinal and count variables", Stata Journal, 6(3), pp. 285-308.

Mroz, T., Guilkey, D. (1992): "Discrete factor approximation for use in simultaneous equation models with both continuous and discrete endogenous variables". Mimeo. Department of Economics, University of North Carolina, Chapel Hill.

Observatorio de personas mayores, (2004): Las personas mayores en España, IMSERSO, Madrid.

Otero, A,. García de Yébenes, M, J,. Rodríguez-Laso, A, y Zunzunegui, M, V, (2003): "Unmet home care needs among community-dwelling elderly people in Spain", Aging Clinical and Experimental Research, 15(3), pp. 234-242.

Pavalko, E, y Artis, J, (1997): "Women's caregiving and paid work: casual relationship in late midlife", Journal of Gerontology: Social Sciences, 52B, pp. s170-s179. 
Pavalko, E, y Henderson, K, (2006): "Combining care work and paid work. Do workplace policies make a difference?" Research on Aging, 28, pp. 359374.

Pearlin, L,. Mullan, J, y Semple, S, (1990): "Caregiving and the stress process: an overview of concepts and their measures", Gerontologist, 30, pp. 583-594.

Pillemer, K, y Suitor, J, J, (2006): "Making choices: a within-family study of care-giver selection", The Gerontologist, 46, pp. 439-448.

Secretaría de Estado de Servicios Sociales, Familias y Discapacidad (2004): Atención a las personas en situación de dependencia en España, Libro Blanco, IMSERSO, Madrid.

Spiess, C, y Schneider, A, (2002): "Midlife caregiving and employment: an analysis of adjustments in work hours and informal care for female employees in Europe", ENEPRI Working Paper No 9, CEPS.

Staiger, D, y Stock, J, (1997): "Instrumental variables regression with weak instruments", Econometrica, 65, pp. 557-586.

Stern, S, (1995): "Estimating family long-term care decisions in the presence of endogenous child characteristics", The Journal of Human Resources, 30, pp. 551-580.

Stevens, A, y Gabbay, J, (1991): "Needs assessment", Health Trends, 23, pp. 20-23.

Stoller, E, P, (1983): "Parental caregiving by adult children", Journal of Marriage and the Family,45, pp. 851-858.

Stone, R,. Cafferata, G, y Sangl, J, (1987): "Caregivers of the frail elderly: a national profile”, The Gerontologist, 27, pp. 616-626.

Terza, J, V,. Kenkel, D, S,. Lin, T, y Sakata, S, (2008): "Care-giver advice as a preventive measure for drinking during pregnancy: zeros, categorical outcome responses and endogeneity", Health Economics, 17, pp. 41-54.

Train, K, (2003): Discrete choice methods with simulation, Cambridge University Press, Cambridge.

Viitanen, T, (2005): "Informal elderly care and female labor participation across Europe”, ENEPRI, Research Report No, 13, Brussels.

Vilaplana, C, (2009): "Necesidades no satisfechas de las personas dependientes en España”, Cuadernos Económicos ICE, 77, pp. 201-231. 
Wolf, D, y Soldo, B, (1994): "Married women's allocation of time to employment and parental care", The Journal of Human Resources, 29, pp. $1259-1276$.

Wooldridge, J, (2003): "Cluster-sample methods in applied econometrics", American Economic Review, 93, pp. 133-138. 


\section{Apéndice}

Tabla 1. Estadísticos descriptivos (con pesos muestrales)

\begin{tabular}{|c|c|c|c|c|}
\hline & \multirow{2}{*}{$\begin{array}{l}\text { No ocupado } \\
\text { antes de ser } \\
\text { cuidador }\end{array}$} & \multicolumn{3}{|c|}{ Ocupado antes de ser cuidador } \\
\hline & & $\begin{array}{c}\text { No problemas } \\
\text { laborales }\end{array}$ & $\begin{array}{l}\text { Sí problemas } \\
\text { laborales }\end{array}$ & $\begin{array}{c}\text { Ha dejado de } \\
\text { trabajar }\end{array}$ \\
\hline \multicolumn{5}{|l|}{ Características del dependiente } \\
\hline \multicolumn{5}{|l|}{ Sexo } \\
\hline Hombre & 0,2240 & 0,2468 & 0,2856 & 0,2645 \\
\hline Mujer & 0,7760 & 0,7532 & 0,7144 & 0,7355 \\
\hline \multicolumn{5}{|l|}{ Edad } \\
\hline $60-69$ & 0,0726 & 0,1127 & 0,1193 & 0,1231 \\
\hline $70-79$ & 0,2192 & 0,4361 & 0,2940 & 0,2703 \\
\hline $80-89$ & 0,5395 & 0,3504 & 0,4755 & 0,4675 \\
\hline 90 y más & 0,1687 & 0,1008 & 0,1112 & 0,1390 \\
\hline Vive solo & 0,2216 & 0,2733 & 0,1831 & 0,0969 \\
\hline \multicolumn{5}{|l|}{ Patologías } \\
\hline Enfermedad mental (a) & 0,2705 & 0,2186 & 0,4076 & 0,3590 \\
\hline Cáncer & 0,0309 & 0,0393 & 0,0851 & 0,0591 \\
\hline Problemas respiratorios & 0,1413 & 0,1254 & 0,2346 & 0,1868 \\
\hline Problemas osteoarticulares & 0,5810 & 0,5393 & 0,5418 & 0,5159 \\
\hline Enfermedad cardiovascular & 0,3211 & 0,2754 & 0,3242 & 0,3651 \\
\hline \multicolumn{5}{|l|}{ Grado de dependencia } \\
\hline Moderado. Nivel 1 & 0,2401 & 0,1610 & 0,2070 & 0,1193 \\
\hline Moderado. Nivel 2 & 0,0870 & 0,0700 & 0,1372 & 0,1568 \\
\hline Severo. Nivel 1 & 0,1050 & 0,0657 & 0,1799 & 0,1943 \\
\hline Severo. Nivel 2 & 0,0280 & 0,0243 & 0,1248 & 0,1125 \\
\hline Grande. Nivel 1 & 0,0471 & 0,0377 & 0,0860 & 0,1465 \\
\hline Grande. Nivel 2 & 0,0083 & 0,0087 & 0,0081 & 0,0105 \\
\hline \multicolumn{5}{|l|}{ Discapacidades } \\
\hline No puede comer por sí solo & 0,1756 & 0,1043 & 0,3194 & 0,3290 \\
\hline No puede utilizar el servicio o baño & 0,1353 & 0,1067 & 0,2338 & 0,2773 \\
\hline No puede asearse después de utilizar el servicio & 0,0678 & 0,0447 & 0,0809 & 0,0917 \\
\hline No controla la micción & 0,0244 & 0,0128 & 0,0323 & 0,0386 \\
\hline No controla la defecación & 0,0316 & 0,0158 & 0,0452 & 0,0507 \\
\hline No le importa la limpieza personal & 0,1838 & 0,1596 & 0,2588 & 0,2712 \\
\hline No puede bañarse/ducharse por sí solo & 0,2016 & 0,1516 & 0,2451 & 0,2569 \\
\hline No puede asearse por sí solo (cara, manos, peinarse) & 0,5760 & 0,4368 & 0,7093 & 0,7339 \\
\hline No puede abrocharse los botones & 0,1338 & 0,1188 & 0,1852 & 0,2053 \\
\hline No puede vestirse/desvestirse & 0,2507 & 0,2002 & 0,4026 & 0,4600 \\
\hline No controla sus medicinas & 0,1940 & 0,1677 & 0,2005 & 0,2067 \\
\hline Sufre caídas o accidentes en el domicilio & 0,1909 & 0,1933 & 0,2440 & 0,2216 \\
\hline No puede utilizar el teléfono & 0,0918 & 0,0659 & 0,1253 & 0,1449 \\
\hline No puede levantarse/acostarse de la cama & 0,3012 & 0,2382 & 0,4619 & 0,5695 \\
\hline Se desorienta por la casa & 0,0757 & 0,0759 & 0,1228 & 0,1331 \\
\hline No puede desplazarse por la casa & 0,1057 & 0,0845 & 0,1852 & 0,2162 \\
\hline No quiere salir de casa & 0,0843 & 0,0944 & 0,1127 & 0,1137 \\
\hline Se desorienta por la calle & 0,0795 & 0,0703 & 0,1373 & 0,1316 \\
\hline No puede utilizar el transporte público & 0,1615 & 0,1492 & 0,1778 & 0,1803 \\
\hline No puede preparar comidas & 0,2946 & 0,2605 & 0,3483 & 0,3713 \\
\hline No puede hacer la compra & 0,1951 & 0,1727 & 0,2098 & 0,2070 \\
\hline No puede realizar otras tareas domésticas & 0,1414 & 0,1327 & 0,1638 & 0,1684 \\
\hline No le importa la limpieza de su ropa & 0,0283 & 0,0246 & 0,0398 & 0,0417 \\
\hline Come cosas que no debe & 0,0260 & 0,0319 & 0,0522 & 0,0397 \\
\hline No puede moverse & 0,0078 & 0,0067 & 0,0147 & 0,0199 \\
\hline Acumula cosas inútiles/no encuentra sus cosas & 0,0129 & 0,0110 & 0,0249 & 0,0182 \\
\hline Agresivo física o verbalmente & 0,0050 & 0,0073 & 0,0120 & 0,0176 \\
\hline No sabe utilizar el dinero & 0,0140 & 0,0099 & 0,0265 & 0,0289 \\
\hline No sabe hacer gestiones & 0,0164 & 0,0133 & 0,0306 & 0,0299 \\
\hline \multicolumn{5}{|l|}{ Ingresos mensuales } \\
\hline Menos de $300 €$ & 0,1453 & 0,1930 & 0,1647 & 0,1992 \\
\hline De 300 a $600 €$ & 0,5573 & 0,5348 & 0,6061 & 0,6397 \\
\hline Más de $600 €$ & 0,0894 & 0,0686 & 0,0763 & 0,0571 \\
\hline
\end{tabular}


Tabla 1 (cont.). Estadísticos descriptivos (con pesos muestrales)

\begin{tabular}{|c|c|c|c|c|}
\hline \multicolumn{5}{|l|}{$\begin{array}{l}\text { Características del cuidador } \\
\text { Sexo }\end{array}$} \\
\hline $\begin{array}{l}\text { Sexo } \\
\text { Hombre }\end{array}$ & 0,1317 & 0,2549 & 0,1521 & 0,0403 \\
\hline Mujer & 0,8683 & 0,7451 & 0,8479 & 0,9597 \\
\hline \multicolumn{5}{|l|}{ Edad } \\
\hline 25-39 & 0,0931 & 0,3155 & 0,2427 & 0,1443 \\
\hline $40-49$ & 0,2739 & 0,4268 & 0,2830 & 0,3239 \\
\hline $50-64$ & 0,6330 & 0,2577 & 0,4744 & 0,5318 \\
\hline \multicolumn{5}{|l|}{ Nivel de estudios } \\
\hline Sin estudios & 0,1252 & 0,0387 & 0,0449 & 0,1759 \\
\hline Primarios & 0,5009 & 0,3245 & 0,3842 & 0,4577 \\
\hline Secundarios & 0,3258 & 0,4139 & 0,4685 & 0,3259 \\
\hline Superiores & 0,0437 & 0,2229 & 0,1024 & 0,0356 \\
\hline \multicolumn{5}{|l|}{ Estado civil } \\
\hline Casado & 0,8603 & 0,6300 & 0,7311 & 0,7767 \\
\hline Separado & 0,0816 & 0,2326 & 0,1740 & 0,1339 \\
\hline Viudo & 0,0296 & 0,0490 & 0,0282 & 0,0591 \\
\hline Separado/divorciado & 0,0199 & 0,0884 & 0,0668 & 0,0253 \\
\hline Sustentador principal del hogar & 0,1970 & 0,4831 & 0,2591 & 0,1008 \\
\hline \multicolumn{5}{|l|}{ Relación con la actividad económica } \\
\hline Ocupado (b) & 0,0000 & 1,0000 & 1,0000 & 0,0000 \\
\hline Empresario sin asalariados & 0,0000 & 0,0944 & 0,1351 & 0,0000 \\
\hline Empresario con asalariados & 0,0000 & 0,0722 & 0,0649 & 0,0000 \\
\hline Profesional & 0,0000 & 0,1500 & 0,1405 & 0,0000 \\
\hline Trabajador cualificado & 0,0000 & 0,4056 & 0,3243 & 0,0000 \\
\hline Trabajador no cualificado & 0,0000 & 0,2556 & 0,3027 & 0,0000 \\
\hline Jubilado & 0,2117 & 0,0000 & 0,0000 & 0,0994 \\
\hline Desempleado & 0,0876 & 0,0000 & 0,0000 & 0,1314 \\
\hline Sus labores & 0,6898 & 0,0000 & 0,0000 & 0,7692 \\
\hline Estudiante & 0,0109 & 0,0000 & 0,0000 & 0,0000 \\
\hline \multicolumn{5}{|l|}{ Situación profesional del sust. principal (distinto del cuidador) } \\
\hline Empresario sin asalariados & 0,1095 & 0,1609 & 0,1460 & 0,1494 \\
\hline Empresario con asalariados & 0,0934 & 0,0750 & 0,0912 & 0,1103 \\
\hline Profesional & 0,0865 & 0,1539 & 0,1311 & 0,0628 \\
\hline Trabajador cualificado & 0,5259 & 0,3871 & 0,4128 & 0,3911 \\
\hline Trabajador no cualificado & 0,1713 & 0,2231 & 0,2092 & 0,2739 \\
\hline \multicolumn{5}{|l|}{ Tiene hijos } \\
\hline Menores de 12 años & 0,1630 & 0,2819 & 0,2260 & 0,2070 \\
\hline De 13 a 17 años & 0,1657 & 0,2481 & 0,2296 & 0,2317 \\
\hline De 18 y más años & 0,5731 & 0,4903 & 0,4898 & 0,6117 \\
\hline \multicolumn{5}{|l|}{$\mathrm{N}^{\circ}$ años de cuidado } \\
\hline Menos de 2 & 0,3437 & 0,4243 & 0,3496 & 0,3373 \\
\hline De 2 a 4 & 0,2197 & 0,2071 & 0,1822 & 0,1668 \\
\hline De 4 a 12 & 0,3390 & 0,3198 & 0,3626 & 0,3588 \\
\hline Más de 12 & 0,0976 & 0,0488 & 0,1056 & 0,1372 \\
\hline \multicolumn{5}{|l|}{ Tamaño del municipio de residencia } \\
\hline Menos de 2.000 habitantes & 0,0984 & 0,0585 & 0,0539 & 0,1168 \\
\hline De 2.001 a 10.000 & 0,1514 & 0,1298 & 0,2187 & 0,2703 \\
\hline De 10.001 a 50.000 & 0,1973 & 0,2474 & 0,2461 & 0,2416 \\
\hline De 50.001 a 500.000 & 0,2561 & 0,1571 & 0,2165 & 0,1680 \\
\hline Capital de provincia & 0,2968 & 0,4071 & 0,2648 & 0,2033 \\
\hline Horas diarias de cuidado informal & 9,3196 & 6,2627 & 11,1772 & 12,4047 \\
\hline$\%$ que dedica menos 1 hora/día & 0,0386 & 0,0454 & 0,0247 & 0,0049 \\
\hline$\%$ que dedica $1-2$ horas/día & 0,1876 & 0,3296 & 0,0914 & 0,0655 \\
\hline$\%$ que dedica 3-5 horas/día & 0,2545 & 0,2981 & 0,2499 & 0,2113 \\
\hline$\%$ que dedica 5-8 horas/día & 0,1815 & 0,1067 & 0,1701 & 0,2228 \\
\hline \% que dedica más de 8 horas/día & 0,3378 & 0,2203 & 0,4639 & 0,4955 \\
\hline Necesidades no satisfechas de Atención formal (NNS) & 0,2675 & 0,2399 & 0,4307 & 0,4457 \\
\hline Atención a Domicilio (c) & 0,2186 & 0,2126 & 0,3961 & 0,3959 \\
\hline Centro de día (c) & 0,1575 & 0,0961 & 0,2649 & 0,3129 \\
\hline $\mathrm{N}$ & 282 & 180 & 394 & 281 \\
\hline
\end{tabular}

(a) Enfermedad mental: toma el valor 1 cuando la persona a la que cuida padece demencia senil, demencia tipo Alzheimer o depresión.

(b) Situación profesional: empresario sin asalariados (empresario agricultor sin trabajadores, empresario no agrario sin asalariados), empresario con trabajadores (empresario agrario con trabajadores, empresario no agrario con trabajadores), profesionales por cuenta ajena (directivos y gerentes, profesionales de la Administración Pública, miembros de las Fuerzas Armadas, profesionales, técnicos y asimilados), trabajadores cualificados (personal administrativo, del sector servicios y comerciales, encargados, contramaestres, capataces, operarios cualificados de establecimientos no agrarios), trabajadores no cualificados (operarios sin cualificación y técnicos de explotaciones agrarias).

(c)Necesidades no Satisfechas: la suma de NNS de Atención a Domicilio y NNS de Centros de Día es mayor que el total de NNS porque hay cuidadores que declaran NNS para ambos servicios. 
Tabla 2. Estimaciones

\begin{tabular}{|c|c|c|c|c|c|c|c|c|c|c|c|c|c|c|}
\hline & \multicolumn{8}{|c|}{$\begin{array}{c}\text { Probit ordenado con } 2 \text { variables endógenas } \\
\text { y sesgo de selección }\end{array}$} & \multicolumn{6}{|c|}{$\begin{array}{c}\text { Probit ordenado con } 1 \text { variable endógena } \\
\text { y sesgo de seleeción }\end{array}$} \\
\hline & \multicolumn{2}{|c|}{ PL } & \multicolumn{2}{|c|}{$\mathrm{W}$} & HI & & NN! & & $\mathrm{PI}$ & & & & NN & \\
\hline & Coef, & $\mathrm{t}$ & Coef, & $\mathrm{t}$ & Coef, & $\mathrm{t}$ & Coef, & $\mathrm{t}$ & Coef, & $\mathrm{t}$ & Coef, & $\mathrm{t}$ & Coef, & $\mathrm{t}$ \\
\hline Receptor de cuidados & & & & & & & & & & & & & & \\
\hline $\begin{array}{l}\text { Hombre } \\
\text { Edad } 70-79\end{array}$ & $\begin{array}{r}-0,103 \\
0175\end{array}$ & $-2,01$ & & & 0,109 & 2,07 & 0,128 & 2,14 & $-0,134$ & $-3,58$ & & & 0,147 & 3,51 \\
\hline $\begin{array}{l}\text { Edad: } 70-79 \\
\text { Edad: } 80-89\end{array}$ & 0,175 & 2,84 & & & $-0,236$ & $-2,15$ & $-0,301$ & $-2,06$ & 0,167 & 2,48 & & & $-0,261$ & $-3,74$ \\
\hline $\begin{array}{l}\text { Edad: } 80-89 \\
\text { Edad: } 90 \text { y más }\end{array}$ & 0,294 & 2,15 & & & $-0,107$ & $-2,41$ & $-0,362$ & $-2,99$ & 0,204 & 3,25 & & & $-0,344$ & $-2,33$ \\
\hline $\begin{array}{l}\text { Edad: } 90 \text { y más } \\
\text { Enfermedad mental }\end{array}$ & 0,347 & 3,64 & & & 0,065 & 2,22 & $-0,152$ & $-2,26$ & 0,337 & 3,23 & & & $-0,179$ & $-1,00$ \\
\hline Enfermedad mental & 0,150 & 3,45 & & & 0,256 & 3,86 & 0,113 & 1,99 & 0,144 & 3,47 & & & 0,155 & 3,61 \\
\hline Cáncer & 0,015 & 3,02 & & & 0,112 & 2,51 & $-0,043$ & $-2,15$ & 0,020 & 2,14 & & & $-0,038$ & $-3,23$ \\
\hline Prob. respiratorios & 0,048 & 3,38 & & & 0,129 & 1,13 & 0,081 & 2,59 & 0,050 & 2,31 & & & 0,092 & 3,83 \\
\hline Prob. osteoarticulares & 0,060 & 3,86 & & & 0,100 & 1,92 & 0,084 & 2,82 & 0,065 & 3,33 & & & 0,074 & 3,86 \\
\hline Enf. cardiovascular & 0,036 & 3,55 & & & 0,023 & 2,36 & $-0,010$ & $-1,90$ & 0,037 & 3,33 & & & $-0,018$ & $-2,20$ \\
\hline Dep. Moderada. Nivel 1 & 0,114 & 2,2 & & & 0,146 & 1,95 & 0,284 & 2,55 & 0,120 & 3,17 & & & 0,285 & 2,21 \\
\hline Dep. Moderada. Nivel 2 & 0,213 & 2,23 & & & 0,486 & 6,41 & 0,238 & 1,78 & 0,222 & 3,94 & & & 0,182 & 3,27 \\
\hline Dep. Severa. Nivel 1 & 0,445 & 2,36 & & & 0,413 & 4,01 & 0,433 & 4,65 & 0,439 & 3,28 & & & 0,409 & 2,95 \\
\hline Dep. Severa. Nivel 2 & 0,507 & 3,48 & & & 0,544 & 8,55 & 0,402 & 2,12 & 0,512 & 3,14 & & & 0,413 & 2,61 \\
\hline Dep. Grande. Nivel 1 & 0,602 & 3,84 & & & 0,505 & 3,77 & 0,412 & 1,87 & 0,679 & 3,12 & & & 0,465 & 2,86 \\
\hline Dep. Grande. Nivel 2 & 0,689 & 2,12 & & & 0,060 & 4,16 & 1,483 & 4,04 & 0,682 & 3,39 & & & 1,522 & 3,07 \\
\hline Ingreso/mes: $300-600 €$ & & & & & & & $-0,020$ & $-2,06$ & & & & & $-0,035$ & $-4,37$ \\
\hline Ingreso/mes: $+600 €$ & & & & & & & $-0,213$ & $-2,85$ & & & & & $-0,181$ & $-3,17$ \\
\hline Vive solo & $-0,029$ & $-2,30$ & & & $-0,419$ & $-4,04$ & 0,141 & 2,64 & $-0,046$ & $-2,46$ & & & 0,109 & 3,98 \\
\hline $\begin{array}{l}\text { Necesidad no satisfecha } \\
\text { Tasa de cobertura }\end{array}$ & 0,470 & 2,17 & & & $-0,898$ & $-2,89$ & & & 0,327 & 7,07 & & & & \\
\hline $\begin{array}{l}\text { Tasa de cobertura } \\
\text { At. a Domicilio }\end{array}$ & & & & & & & $-0,005$ & $-2,25$ & & & & & $-0,007$ & $-3,16$ \\
\hline Centro Día & & & & & & & $-0,102$ & $-2,03$ & & & & & $-0,106$ & $-2,64$ \\
\hline $\begin{array}{l}\% \text { At. Dom. Cuid. person. } \\
\text { Cuidador informal }\end{array}$ & & & & & & & $-0,005$ & $-3,20$ & & & & & $-0,006$ & $-2,99$ \\
\hline Hombre & $-0,367$ & $-2,67$ & $-0,106$ & $-2,68$ & 0,258 & 2,51 & $-0,057$ & $-2,18$ & $-0,378$ & $-2,77$ & $-0,093$ & $-2,70$ & $-0,059$ & $-2,06$ \\
\hline $\begin{array}{l}\text { Edad dep-Edad cuidador } \\
\text { Mismo sexo cuidador-dep. }\end{array}$ & & & & & $\begin{array}{r}-0,001 \\
0,127\end{array}$ & $\begin{array}{r}-2,14 \\
2,01\end{array}$ & $\begin{array}{r}0,005 \\
-0,134\end{array}$ & $\begin{array}{r}0,58 \\
-2,35\end{array}$ & & & & & $\begin{array}{r}0,006 \\
-0,156\end{array}$ & $\begin{array}{r}2,02 \\
-2,11\end{array}$ \\
\hline Edad: $40-49$ & 0,280 & 2,46 & $-0,364$ & $-2,82$ & $-0,090$ & $-3,67$ & 0,053 & 2,21 & 0,290 & 3,83 & $-0,363$ & $-2,36$ & $\begin{array}{r}0,053 \\
0,053\end{array}$ & $\begin{array}{r}2,11 \\
2,03\end{array}$ \\
\hline Edad: 5 & 0,391 & 2,29 & $-0,497$ & $-3,67$ & $-0,049$ & $-3,25$ & $-0,031$ & $-2,06$ & 0,297 & 2,50 & $-0,487$ & $-3,12$ & $-0,046$ & $-2,45$ \\
\hline Estudios primarios & $-0,674$ & $-2,91$ & 0,023 & 2,15 & $-0,101$ & $-3,6$ & 0,298 & 2,23 & $-0,723$ & $-4,56$ & 0,018 & 3,13 & 0,337 & 2,20 \\
\hline Estudios secundarios & $-0,675$ & $-2,12$ & 0,232 & 2,54 & $-0,196$ & $-3,94$ & 0,175 & 2,70 & $-0,640$ & $-4,02$ & 0,237 & 3,58 & 0,197 & 3,24 \\
\hline Estudios superiores & $-0,933$ & $-2,02$ & 0,447 & 2,10 & $-0,180$ & $-3,76$ & 0,375 & 2,00 & $-0,958$ & $-4,69$ & 0,459 & 2,07 & 0,350 & 3,61 \\
\hline Situación profesional & & & & & & & & & & & & & & \\
\hline Empr. sin asal. & $-1,187$ & $-3,93$ & & & 0,069 & 4,45 & 0,030 & 2,13 & $-1,093$ & $-5,80$ & & & 0,042 & 3,23 \\
\hline Empr. con asal. & $-1,300$ & $-3,92$ & & & $-0,609$ & $-2,66$ & $-0,153$ & $-2,33$ & $-0,923$ & $-4,05$ & & & $-0,176$ & $-2,32$ \\
\hline Profesional & $-1,435$ & $-3,09$ & & & 0,064 & 4,27 & $-0,388$ & $-2,89$ & $-1,198$ & $-4,49$ & & & $-0,379$ & $-3,33$ \\
\hline Trab. cualificado & $-1,060$ & $-3,47$ & & & $-0,010$ & $-4,11$ & $-0,043$ & $-2,23$ & $-0,949$ & $-5,91$ & & & $-0,058$ & $-3,07$ \\
\hline Trab. no cualificado & $-1,085$ & $-3,41$ & & & $-0,037$ & $-4,36$ & $-0,414$ & $-2,39$ & $-0,913$ & $-4,41$ & & & $-0,426$ & $-2,38$ \\
\hline Sustentador principal & $-0,123$ & $-3,52$ & & & $-0,221$ & $-4,52$ & $-0,025$ & $-2,09$ & $-0,101$ & $-2,57$ & & & $-0,031$ & $-3,24$ \\
\hline $\begin{array}{c}\text { Años prestando cui } \\
\text { Menos de } 2\end{array}$ & $-0,288$ & $-2,48$ & & & $-0,226$ & $-1,88$ & 0,276 & 1,78 & $-0,317$ & $-2,29$ & & & 0,242 & 3,56 \\
\hline De 2 a 4 & $-0,287$ & $-2,78$ & & & $-0,258$ & $-1,83$ & $-0,090$ & $-2,06$ & $-0,152$ & $-3,02$ & & & $-0,082$ & $-2,49$ \\
\hline De 4 a 12 & $-0,263$ & $-2,54$ & & & $-0,072$ & $-4,46$ & 0,174 & 2,15 & $-0,293$ & $-2,15$ & & & 0,167 & 3,95 \\
\hline Horas diarias de CI (log.) & 0,197 & 3,38 & & & & & 0,325 & 2,58 & 0,193 & 2,25 & & & 0,327 & 2,45 \\
\hline Relación cuidador-depen. & & & & & & & & & & & & & & \\
\hline Esposo/ & & & & & 1,312 & 7,36 & 0,599 & 1,97 & & & & & 0,605 & 2,03 \\
\hline & & & & & 0,412 & 4,33 & 0,179 & 1,68 & & & & & 0,167 & 1,79 \\
\hline Hijo/a político & & & & & 0,340 & 2,35 & 0,131 & 1,57 & & & & & 0,143 & 1,81 \\
\hline Estado civil & & & & & & & & & & & & & & \\
\hline Casado & & & $-0,399$ & $\begin{array}{l}-2,42 \\
-250\end{array}$ & & & & & & & $-0,410$ & $-2,46$ & & \\
\hline Soltero & & & 0,106 & 2,52 & & & & & & & 0,103 & 3,81 & & \\
\hline $\begin{array}{l}\text { Viudo } \\
\text { Sit }\end{array}$ & & & 0,154 & 2,35 & & & & & & & 0,144 & 3,49 & & \\
\hline Sit. Prof. Sustent. Princ. & & & 0.362 & & & & & & & & & & & \\
\hline Emi & & & $\begin{array}{l}0,362 \\
0,350\end{array}$ & 2,87 & & & & & & & 0,340 & 3,46 & & \\
\hline Em & & & 0,350 & 2,8 & & & & & & & 0,378 & 3,62 & & \\
\hline Profesional & & & 0,283 & 3, & & & & & & & 0,270 & 3,87 & & \\
\hline Trab. cualificado & & & 0,004 & 2,0 & & & & & & & 0,008 & 0,87 & & \\
\hline $\begin{array}{l}\text { Trab. no cualificado } \\
\text { Tisa }\end{array}$ & & & 0,370 & 2,87 & & & & & & & 0,380 & 1,88 & & \\
\hline Tiene hijos en el ho & & & & 1,39 & & & & & & & & & & \\
\hline Menor & & & 0,069 & 1,39 & & & & & & & 0,079 & 0 , & & \\
\hline $\begin{array}{l}\text { De } 13 \text { a } 17 \text { años } \\
\text { Tamaño del municipio }\end{array}$ & & & 0,214 & 1,8 & & & & & & & 0,221 & 1,88 & & \\
\hline $\begin{array}{l}\text { Tamaño del municipio } \\
\text { Menos de } 2.000 \text { hab. }\end{array}$ & & & $-0,091$ & $-2,36$ & & & 0,233 & 3,06 & & & 0099 & 218 & 0,236 & \\
\hline De 2.001 a 10.000 & & & 0,401 & 2,78 & & & $-0,104$ & $-2,16$ & & & $\begin{array}{r}-0,092 \\
0,455\end{array}$ & $\begin{array}{r}-10 \\
3,63\end{array}$ & $\begin{array}{r}0,200 \\
-0,111\end{array}$ & $\begin{array}{r}0,01 \\
-3,33 \\
-3\end{array}$ \\
\hline De 10.001 a 50.000 & & & 0,193 & 1,97 & & & $-0,030$ & $-2,01$ & & & 0,224 & 3,90 & $-0,028$ & $-3,91$ \\
\hline De 50. & & & $-0,135$ & $-2,71$ & & & $-0,024$ & 2,25 & & & $-0,108$ & $-2,94$ & 0,021 & 3,88 \\
\hline Constante & & & 0,964 & 1,88 & 1,934 & 5,97 & $-0,626$ & $-1,77$ & & & 0,944 & $\begin{array}{l}3,98 \\
3,98\end{array}$ & $-0,657$ & $-2,97$ \\
\hline$\rho($ & $-0,581$ & $-2,22$ & $\mathrm{~N}$ & & & & & 1,137 & & & $-0,628$ & $-2,68$ & & 1137 \\
\hline NS) & 0,387 & 1,93 & Log pse & adolike & ihood & & -3321 & 9443 & $\rho(\mathrm{PL}-\mathrm{N}$ & & 0,342 & 3,86 & $\mathrm{LR} \chi^{2}$ & \\
\hline$\rho($ PL-H & 0 , & 1,43 & AIC & & & & 685 & 9,889 & $\rho(\mathrm{W}-\mathrm{NN}$ & & 0,199 & 2,06 & 579,11 & ;0000) \\
\hline$\rho(\mathrm{W}-\mathrm{HI})$ & 0,149 & 2,08 & $\mathrm{BIC}$ & & & & & 3,793 & $\tau_{1}$ & & $-2,505$ & $-3,21$ & & \\
\hline$\rho(W-N N S)$ & 0,249 & 3,09 & $\mathrm{LR} \chi^{2}(1$ & 37) $=$ & 667,04 & & & 0000) & & & $-1,602$ & $-2,37$ & & \\
\hline$\rho$ (NNS-HI) & 0,299 & 1,30 & & & & & & & Log ps,1 & ikelih & -181 & 2575 & & \\
\hline . & $-2,672$ & $-6,34$ & & & & & & & & & & & & \\
\hline & $-1,756$ & $-3,23$ & & & & & & & BI & & & 35,093 & & \\
\hline
\end{tabular}

Variables omitidas: persona que recibe cuidados (edad 60-69 años, calificada como "no dependiente", ingresos/mes menos de 300€, no vive solo), cuidador (edad menos de 40 años, sin estudios, separado/divorciado, no ocupado, sustentador principal no ocupado, hijos en el hogar de 18 y más años, más de 12 años prestando cuidados, reside en capital de provincia). Estimación por Máxima Verosimilitud Simulada (secuencia de Hammersley para la simulación GHK y técnica de DavidonFletcher-Powerll para el cálculo de log-likelihood en cada simulación). Utilización de pesos muestrales y cluster por comunidad autónoma. 
Tabla 3. Test de diagnóstico

\begin{tabular}{|c|c|c|c|c|c|c|}
\hline & \multicolumn{4}{|c|}{$\begin{array}{l}\text { Probit ordenado con dos variables endógenas y sesgo de } \\
\text { selección }\end{array}$} & \multicolumn{2}{|c|}{$\begin{array}{l}\text { Probit ordenado con una variable } \\
\text { endógena y sesgo de selección }\end{array}$} \\
\hline & \multicolumn{2}{|l|}{$\mathrm{HI}$} & \multicolumn{2}{|c|}{ NNS } & \multicolumn{2}{|c|}{ NNS } \\
\hline R2 parcial de los instruí. excluidos & \multicolumn{2}{|c|}{0,4315} & \multicolumn{2}{|c|}{0,4281} & \multicolumn{2}{|c|}{0,4127} \\
\hline Estadístico J de Hansen & $\chi^{2}(4)=12,295$ & $(0,0153)$ & $\chi^{2}(8)=31,915$ & $(0,0001)$ & $\chi^{2}(8)=29,75$ & $(0,0002)$ \\
\hline Test Wald Anderson-Rubin & $\mathrm{F}(5,15)=2,44$ & $(0,0333)$ & $F(9,743)=3,55$ & $(0,0002)$ & $F(9,742)=3,34$ & $(0,0005)$ \\
\hline Estadístico $\mathrm{F}$ de instrum, exlcuidos & $F(5,15)=14,07$ & $(0,0000)$ & $F(9,743)=2,39$ & $(0,0115)$ & $F(9,742)=2,40$ & $(0,0111)$ \\
\hline Estadístico rk Kleibergen-Paap & $\chi^{2}(5)=19,316$ & $(0,0017)$ & $\chi^{2}(9)=19,78$ & $(0,0173)$ & $F(9,742)=19,61$ & $(0,0205)$ \\
\hline Estadístico Wald rk Kleibergen-Paap & $\chi^{2}(5)=32,76$ & $(0,0000)$ & $\chi^{2}(9)=19,26$ & $(0,0230)$ & $F(9,742)=21,13$ & $(0,0121)$ \\
\hline Test & \multicolumn{2}{|c|}{ Autor } & \multicolumn{4}{|c|}{ Hipótesis nula } \\
\hline Estadístico J de Hansen & \multicolumn{2}{|l|}{ Hansen (1982) } & \multicolumn{4}{|c|}{ Ho: Instrumentos exógenos y adecuadamente excluidos } \\
\hline Test Wald Anderson-Rubin & \multicolumn{2}{|c|}{ Anderson and Rubin (1949) } & \multicolumn{4}{|c|}{ Ho: Coeficiente del regresor endógeno es igual a cero } \\
\hline Estadístico $\mathrm{F}$ de instrumentos exl & \multicolumn{2}{|c|}{ Staiger and Stock (1997) } & \multicolumn{4}{|c|}{ Ho: VI excluidas son significativas conjuntamente } \\
\hline Estadístico LM rk Kleibergen-Paap & \multicolumn{2}{|c|}{ Kleibergen and Paap (2006) } & \multicolumn{4}{|c|}{ Ho: Modelo infraidentificado } \\
\hline Estadístico Warld rk Kleibergen-Paap & \multicolumn{2}{|c|}{ Kleibergen and Paap (2006) } & \multicolumn{4}{|c|}{$\begin{array}{l}\text { Ho: Modelo infraidentificado } \\
\text { Ho: Modelo infraidentificado }\end{array}$} \\
\hline
\end{tabular}

Tabla 4. Probabilidades predichas

\begin{tabular}{|c|c|c|c|c|c|c|c|c|c|}
\hline & \multicolumn{2}{|c|}{$\mathrm{PL}=1$} & \multicolumn{2}{|c|}{$\mathrm{PL}=2$} & \multicolumn{2}{|c|}{$\mathrm{PL}=3$} & \multicolumn{3}{|c|}{ Tasa de variación } \\
\hline & Coef, & Std,dev, & Coef, & Std,dev, & Coef, & Std, dev, & $\begin{array}{c}\mathrm{PL}=1 \\
\text { respecto } \\
\mathrm{PL}=2\end{array}$ & $\begin{array}{c}\mathrm{PL}=1 \\
\text { respecto } \\
\mathrm{PL}=3\end{array}$ & $\begin{array}{c}\mathrm{PL}=2 \\
\text { respecto } \\
\mathrm{PL}=3\end{array}$ \\
\hline \multicolumn{10}{|l|}{ Eq. [Prob. laborales] } \\
\hline \multicolumn{10}{|l|}{ Sexo cuidador } \\
\hline Hombre & 0,3688 & 0,0832 & 0,3308 & 0,0946 & 0,3004 & 0,1225 & 11,50 & 22,79 & 10,12 \\
\hline Mujer & 0,1737 & 0,0606 & 0,2885 & 0,1309 & 0,5378 & 0,1395 & $-39,78$ & $-67,70$ & $-46,36$ \\
\hline \multicolumn{10}{|l|}{ Edad cuidador } \\
\hline Menos de 40 & 0,2893 & 0,0767 & 0,3311 & 0,1027 & 0,3796 & 0,1299 & $-12,64$ & $-23,79$ & $-12,76$ \\
\hline 40 a 49 & 0,2191 & 0,0698 & 0,3014 & 0,1251 & 0,4796 & 0,1448 & $-27,31$ & $-54,32$ & $-37,16$ \\
\hline 50 a 64 & 0,1479 & 0,0557 & 0,2740 & 0,1344 & 0,5781 & 0,1362 & $-46,02$ & $-74,41$ & $-52,60$ \\
\hline \multicolumn{10}{|l|}{ Situación profesional } \\
\hline Emp. con asalar. & 0,2474 & 0,0628 & 0,3722 & 0,0581 & 0,3804 & 0,0954 & $-33,53$ & $-34,96$ & 32,78 \\
\hline Emp. sin asalar. & 0,3232 & 0,0772 & 0,3398 & 0,0831 & 0,337 & 0,1229 & $-4,89$ & $-4,09$ & 0,82 \\
\hline Profesional & 0,4734 & 0,081 & 0,3823 & 0,0899 & 0,1443 & 0,0982 & 23,83 & 228,07 & 58,65 \\
\hline Trabajador cualif. & 0,3439 & 0,0723 & 0,3486 & 0,0645 & 0,3075 & 0,1153 & $-1,35$ & 11,84 & 13,41 \\
\hline Trabajador no cualif. & 0,2888 & 0,0589 & 0,3631 & 0,0757 & 0,3481 & 0,1136 & $-20,46$ & $-17,04$ & 4,29 \\
\hline \multicolumn{10}{|l|}{ Patologías dependiente } \\
\hline Enf. mental & 0,1045 & 0,0690 & 0,2778 & 0,1316 & 0,6117 & 0,1456 & $-62,38$ & $-83,08$ & $-54,59$ \\
\hline Cancer & 0,1932 & 0,0644 & 0,3042 & 0,1127 & 0,5026 & 0,1353 & $-36,50$ & $-61,57$ & $-39,48$ \\
\hline Prob. Respiratorios & 0,1863 & 0,0681 & 0,2820 & 0,1296 & 0,5318 & 0,1446 & $-33,95$ & $-64,97$ & $-46,97$ \\
\hline Prob. Osteoartic. & 0,1973 & 0,0677 & 0,2908 & 0,1284 & 0,5120 & 0,1444 & $-32,16$ & $-61,47$ & $-43,20$ \\
\hline Enf. Caridiovasc. & 0,1726 & 0,0627 & 0,2843 & 0,1276 & 0,5431 & 0,1395 & $-39,31$ & $-68,22$ & $-47,64$ \\
\hline \multicolumn{10}{|l|}{ Grado de dependencia } \\
\hline No dependiente & 0,5647 & 0,0736 & 0,2289 & 0,1032 & 0,2064 & 0,1305 & 146,70 & 173,59 & 10,90 \\
\hline Moderado. Nivel 1 & 0,4196 & 0,0695 & 0,2255 & 0,1218 & 0,3549 & 0,1419 & 86,08 & 18,23 & $-36,46$ \\
\hline Moderado. Nivel 2 & 0,3871 & 0,0573 & 0,2779 & 0,1367 & 0,335 & 0,1398 & 39,29 & 15,55 & $-17,04$ \\
\hline Severo. Nivel 1 & 0,2484 & 0,0583 & 0,3674 & 0,1329 & 0,3842 & 0,1389 & $-32,39$ & $-35,35$ & $-4,37$ \\
\hline Severo. Nivel 2 & 0,1547 & 0,0641 & 0,3499 & 0,1474 & 0,4954 & 0,1512 & $-55,79$ & $-68,77$ & $-29,37$ \\
\hline Grande. Nivel 1 & 0,1187 & 0,0497 & 0,2566 & 0,1346 & 0,6247 & 0,1279 & $-53,74$ & $-81,00$ & $-58,92$ \\
\hline Grande. Nivel 2 & 0,0929 & 0,0369 & 0,2552 & 0,1178 & 0,6519 & 0,112 & $-63,60$ & $-85,75$ & $-60,85$ \\
\hline \multicolumn{10}{|l|}{ Horas diarias CI } \\
\hline Menos de $1(0,5 \mathrm{~h})$ & 0,3088 & 0,0925 & 0,2971 & 0,1182 & 0,3941 & 0,1518 & 3,94 & $-21,64$ & $-24,62$ \\
\hline De 1 a $2(1,5 \mathrm{~h})$ & 0,3042 & 0,0747 & 0,3395 & 0,0980 & 0,3563 & 0,1253 & $-10,39$ & $-14,62$ & $-4,72$ \\
\hline De 3 a 5 (4h.) & 0,2270 & 0,0680 & 0,3147 & 0,1172 & 0,4583 & 0,1386 & $-27,87$ & $-50,47$ & $-31,33$ \\
\hline De 5 a $8(6,5 \mathrm{~h})$. & 0,1739 & 0,0602 & 0,2920 & 0,1303 & 0,5341 & 0,1378 & $-40,46$ & $-67,44$ & $-45,32$ \\
\hline Más de 8 & 0,1456 & 0,0573 & 0,2664 & 0,1359 & 0,5880 & 0,1381 & $-45,35$ & $-75,24$ & $-54,68$ \\
\hline \multicolumn{10}{|l|}{ Años de cuidado } \\
\hline Menos de 2 & 0,2171 & 0,0710 & 0,2995 & 0,1269 & 0,4834 & 0,1442 & $-27,50$ & $-55,09$ & $-38,06$ \\
\hline De 2 a 4 & 0,2049 & 0,0682 & 0,2968 & 0,1203 & 0,4983 & 0,1429 & $-30,98$ & $-58,89$ & $-40,43$ \\
\hline De 4 a 12 & 0,1920 & 0,0653 & 0,2945 & 0,1277 & 0,5135 & 0,1412 & $-34,83$ & $-62,62$ & $-42,65$ \\
\hline Más de 12 & 0,1357 & 0,0534 & 0,2643 & 0,1414 & 0,5999 & 0,1360 & $-48,65$ & $-77,38$ & $-55,94$ \\
\hline
\end{tabular}


Tabla 4 (cont.). Probabilidades predichas

\begin{tabular}{|c|c|c|c|c|c|c|c|c|c|}
\hline & \multicolumn{2}{|c|}{$\mathrm{PL}=1$} & \multicolumn{2}{|c|}{$\mathrm{PL}=2$} & \multicolumn{2}{|c|}{$\mathrm{PL}=3$} & \multicolumn{3}{|c|}{ Tasa de variación } \\
\hline & Coef, & Std,dev, & Coef, & Std,dev, & Coef, & Std,dev, & $\begin{array}{c}\mathrm{PL}=1 \\
\text { respecto } \\
\mathrm{PL}=2\end{array}$ & $\begin{array}{c}\mathrm{PL}=1 \\
\text { respecto } \\
\mathrm{PL}=3\end{array}$ & $\begin{array}{c}\mathrm{PL}=2 \\
\text { respecto } \\
\mathrm{PL}=3\end{array}$ \\
\hline \multicolumn{10}{|c|}{ Submuestras de cuidadores caracterizados por: } \\
\hline \multicolumn{10}{|l|}{ Relación de parentesco } \\
\hline Esposo & 0,4570 & 0,2340 & 0,3921 & 0,1297 & 0,1508 & 0,0556 & 16,55 & 202,97 & 159,95 \\
\hline Esposa & 0,0733 & 0,0344 & 0,2061 & 0,1091 & 0,7206 & 0,1604 & $-64,45$ & $-89,83$ & $-71,39$ \\
\hline Hijo & 0,3352 & 0,0810 & 0,3355 & 0,0679 & 0,3293 & 0,1639 & $-0,07$ & 1,80 & 1,87 \\
\hline Hija & 0,1622 & 0,0568 & 0,2883 & 0,0934 & 0,5495 & 0,1806 & $-43,72$ & $-70,47$ & $-47,54$ \\
\hline Yerno & 0,4663 & 0,0611 & 0,3582 & 0,0424 & 0,1755 & 0,0891 & 30,19 & 165,77 & 104,15 \\
\hline Nuera & 0,1795 & 0,0635 & 0,2811 & 0,0966 & 0,5394 & 0,1956 & $-36,15$ & $-66,73$ & $-47,89$ \\
\hline \multicolumn{10}{|l|}{ Hombre cuida de: } \\
\hline Hombre dependiente & 0,3941 & 0,0802 & 0,3411 & 0,0634 & 0,2648 & 0,1468 & 15,52 & 48,80 & 28,81 \\
\hline Mujer dependiente & 0,3627 & 0,0842 & 0,3283 & 0,0687 & 0,3090 & 0,1673 & 10,49 & 17,36 & 6,22 \\
\hline \multicolumn{10}{|l|}{ Mujer cuida de: } \\
\hline Hombre dependiente & 0,1702 & 0,0623 & 0,2807 & 0,0941 & 0,5491 & 0,1886 & $-39,36$ & $-69,00$ & $-48,87$ \\
\hline Mujer dependiente & 0,1750 & 0,0600 & 0,2913 & 0,0932 & 0,5338 & 0,1851 & $-39,92$ & $-67,22$ & $-45,43$ \\
\hline \multicolumn{10}{|l|}{ Edad depen.-Edad cuid. } \\
\hline-3 a 10 años & 0,1070 & 0,0491 & 0,2433 & 0,0979 & 0,6497 & 0,1688 & $-56,00$ & $-83,52$ & $-62,56$ \\
\hline 11 a 20 años & 0,2086 & 0,0709 & 0,2792 & 0,0993 & 0,5122 & 0,2122 & $-25,28$ & $-59,28$ & $-45,50$ \\
\hline 21 a 30 años & 0,1705 & 0,0594 & 0,2954 & 0,0890 & 0,5341 & 0,1770 & $-42,30$ & $-68,09$ & $-44,69$ \\
\hline 31 a 40 años & 0,2019 & 0,0698 & 0,2868 & 0,0945 & 0,5113 & 0,1975 & $-29,58$ & $-60,51$ & $-43,92$ \\
\hline 41 a 50 años & 0,2274 & 0,0678 & 0,3154 & 0,0848 & 0,4571 & 0,1854 & $-27,89$ & $-50,24$ & $-31,00$ \\
\hline Más de 50 años & 0,3290 & 0,0763 & 0,3395 & 0,0659 & 0,3315 & 0,1669 & $-3,09$ & $-0,74$ & 2,42 \\
\hline \multicolumn{10}{|c|}{$\%$ At. Dom. Dedicado a cuidados personales } \\
\hline Menos 25\% & 0,1806 & 0,0648 & 0,2885 & 0,0890 & 0,5309 & 0,1855 & $-37,41$ & $-65,98$ & $-45,64$ \\
\hline $26 \%-50 \%$ & 0,2030 & 0,0755 & 0,2780 & 0,0931 & 0,5190 & 0,1996 & $-26,97$ & $-60,89$ & $-46,44$ \\
\hline $51 \%-75 \%$ & 0,3032 & 0,0698 & 0,3512 & 0,0594 & 0,3456 & 0,1602 & $-13,68$ & $-12,27$ & 1,63 \\
\hline Más del 75\% & 0,2434 & 0,0663 & 0,3287 & 0,0841 & 0,4279 & 0,1793 & $-25,96$ & $-43,12$ & $-23,18$ \\
\hline \multicolumn{10}{|l|}{ NNS } \\
\hline Total & 0,0501 & 0,0223 & 0,1859 & 0,0937 & 0,7641 & 0,1295 & $-73,07$ & $-93,45$ & $-75,67$ \\
\hline \multicolumn{10}{|l|}{ Solicita. no recibe: } \\
\hline At. Domicilio & 0,0390 & 0,0175 & 0,1747 & 0,0813 & 0,7863 & 0,1090 & $-77,70$ & $-95,04$ & $-77,78$ \\
\hline C. Día & 0,0528 & 0,0236 & 0,1830 & 0,1060 & 0,7642 & 0,1430 & $-71,16$ & $-93,09$ & $-76,06$ \\
\hline \multicolumn{10}{|l|}{ Insuficiente calidad } \\
\hline At. Domicilio & 0,0621 & 0,0261 & 0,2041 & 0,0996 & 0,7338 & 0,1426 & $-69,57$ & $-91,54$ & $-72,19$ \\
\hline \multicolumn{10}{|l|}{ Tamaño municipio } \\
\hline Menos de 2.000 hab. & 0,1416 & 0,0498 & 0,2967 & 0,1226 & 0,5617 & 0,1197 & $-52,28$ & $-74,79$ & $-47,17$ \\
\hline De 2.001 a 10.000 & 0,1882 & 0,0653 & 0,2865 & 0,1327 & 0,5253 & 0,1459 & $-34,32$ & $-64,17$ & $-45,45$ \\
\hline De 10.001 a 50.000 & 0,1879 & 0,0614 & 0,2972 & 0,1293 & 0,5149 & 0,1412 & $-36,76$ & $-63,50$ & $-42,28$ \\
\hline De 50.001 a 500.000 & 0,1826 & 0,0681 & 0,2810 & 0,1364 & 0,5364 & 0,1438 & $-35,03$ & $-65,97$ & $-47,62$ \\
\hline Capital de provincia & 0,2381 & 0,0744 & 0,3032 & 0,1184 & 0,4587 & 0,1453 & $-21,47$ & $-48,08$ & $-33,89$ \\
\hline \multicolumn{10}{|l|}{ CC.AA de residencia } \\
\hline Andalucía & 0,1755 & 0,0620 & 0,2850 & 0,1356 & 0,5394 & 0,1425 & $-38,42$ & $-67,46$ & $-47,16$ \\
\hline Aragón & 0,2448 & 0,0750 & 0,3265 & 0,1040 & 0,4287 & 0,1271 & $-25,01$ & $-42,89$ & $-23,85$ \\
\hline Asturias & 0,2473 & 0,0647 & 0,3467 & 0,1040 & 0,4060 & 0,1213 & $-28,68$ & $-39,10$ & $-14,61$ \\
\hline Baleares & 0,2129 & 0,0624 & 0,3256 & 0,1354 & 0,4615 & 0,1414 & $-34,61$ & $-53,87$ & $-29,45$ \\
\hline Canarias & 0,1667 & 0,0628 & 0,2930 & 0,1341 & 0,5403 & 0,1324 & $-43,09$ & $-69,14$ & $-45,78$ \\
\hline Cantabria & 0,2791 & 0,0793 & 0,3054 & 0,1197 & 0,4155 & 0,1595 & $-8,59$ & $-32,82$ & $-26,50$ \\
\hline Cataluña & 0,2326 & 0,0667 & 0,3255 & 0,1189 & 0,4419 & 0,1333 & $-28,54$ & $-47,36$ & $-26,34$ \\
\hline C. Mancha & 0,1427 & 0,0629 & 0,2644 & 0,1170 & 0,5929 & 0,1320 & $-46,02$ & $-75,93$ & $-55,42$ \\
\hline C. León & 0,2090 & 0,0624 & 0,3118 & 0,1256 & 0,4791 & 0,1411 & $-32,96$ & $-56,37$ & $-34,92$ \\
\hline C. Valenciana & 0,1828 & 0,0670 & 0,2800 & 0,1292 & 0,5372 & 0,1451 & $-34,73$ & $-65,98$ & $-47,88$ \\
\hline Extremadura & 0,1790 & 0,0537 & 0,3268 & 0,0992 & 0,4943 & 0,1177 & $-45,24$ & $-63,79$ & $-33,88$ \\
\hline Galicia & 0,2423 & 0,0746 & 0,2985 & 0,1243 & 0,4591 & 0,1519 & $-18,83$ & $-47,22$ & $-34,98$ \\
\hline Madrid & 0,1406 & 0,0672 & 0,2352 & 0,1360 & 0,6242 & 0,1449 & $-40,24$ & $-77,48$ & $-62,32$ \\
\hline Murcia & 0,2180 & 0,0838 & 0,2612 & 0,1204 & 0,5208 & 0,1618 & $-16,53$ & $-58,14$ & $-49,86$ \\
\hline Navarra & 0,3477 & 0,0635 & 0,3538 & 0,0985 & 0,2985 & 0,1328 & $-1,73$ & 16,49 & 18,54 \\
\hline País Vasco & 0,2386 & 0,0739 & 0,3069 & 0,1238 & 0,4544 & 0,1451 & $-22,25$ & $-47,48$ & $-32,45$ \\
\hline
\end{tabular}

Para las categorías relación de parentesco, diferencia de edad entre cuidador y dependiente, diferencia de sexo entre cuidador y dependiente, tamaño del municipio de residencia, comunidad autónoma de residencia y necesidades no satisfechas las probabilidades se refieren a la media de las probabilidades predichas para el conjunto de individuos que cumplen una determinada característica. 
Tabla 5. Simulación del efecto derivado de la solución de las NNS

\begin{tabular}{|c|c|c|c|c|c|c|c|c|c|}
\hline & \multicolumn{3}{|c|}{$\begin{array}{l}\text { Probabilidades predichas } \\
\text { cuando hay NNS } \\
\text { (situación actual) }\end{array}$} & \multicolumn{3}{|c|}{$\begin{array}{c}\text { Probabilidades predichas si se } \\
\text { solucionan las NNS } \\
\text { (situación simulada) }\end{array}$} & \multicolumn{3}{|c|}{$\begin{array}{c}\text { Tasas de variación situación } \\
\text { simulada respecto a } \\
\text { situación real }\end{array}$} \\
\hline & $\mathrm{PL}=1$ & $\mathrm{PL}=2$ & $\mathrm{PL}=3$ & $\mathrm{PL}=1$ & $\mathrm{PL}=2$ & $\mathrm{PL}=3$ & $\mathrm{PL}=1$ & $\mathrm{PL}=2$ & $\mathrm{PL}=3$ \\
\hline Andalucía & 0,0488 & 0,1657 & 0,7855 & 0,2572 & 0,3319 & 0,4109 & 427,00 & 100,37 & $-47,69$ \\
\hline Std. Dev. & 0,0145 & 0,0719 & 0,2137 & 0,1164 & 0,0651 & 0,1963 & & & \\
\hline Aragón & 0,0509 & 0,2198 & 0,7292 & 0,3280 & 0,3842 & 0,2877 & 544,08 & 74,79 & $-60,54$ \\
\hline Std. Dev. & 0,0091 & 0,0586 & 0,1620 & 0,0894 & 0,0405 & 0,1452 & & & \\
\hline Asturias & 0,0424 & 0,1828 & 0,7748 & 0,2759 & 0,3828 & 0,3413 & 550,90 & 109,42 & $-55,95$ \\
\hline Std. Dev. & 0,0118 & 0,0627 & 0,1834 & 0,0992 & 0,0400 & 0,1402 & & & \\
\hline Baleares & 0,1113 & 0,2730 & 0,6156 & 0,4407 & 0,3297 & 0,2295 & 295,95 & 20,77 & $-62,72$ \\
\hline Std. Dev. & 0,0228 & 0,0855 & 0,2842 & 0,1489 & 0,0913 & 0,1799 & & & \\
\hline Canarias & 0,0407 & 0,1710 & 0,7883 & 0,2596 & 0,3729 & 0,3675 & 538,04 & 118,12 & $-53,38$ \\
\hline Std. Dev. & 0,0125 & 0,0605 & 0,1804 & 0,0977 & 0,0549 & 0,1512 & & & \\
\hline Cantabria & 0,0348 & 0,1679 & 0,7973 & 0,2516 & 0,3859 & 0,3625 & 622,97 & 129,86 & $-54,54$ \\
\hline Std. Dev. & 0,0105 & 0,0613 & 0,1747 & 0,0953 & 0,0340 & 0,1403 & & & \\
\hline Cataluña & 0,0371 & 0,1680 & 0,7949 & 0,2533 & 0,3780 & 0,3687 & 583,17 & 124,91 & $-53,61$ \\
\hline Std. Dev. & 0,0111 & 0,0566 & 0,1667 & 0,0906 & 0,0556 & 0,1454 & & & \\
\hline C. Mancha & 0,0481 & 0,1863 & 0,7657 & 0,2838 & 0,3648 & 0,3514 & 490,28 & 95,84 & $-54,10$ \\
\hline Std. Dev. & 0,0133 & 0,0640 & 0,1903 & 0,1034 & 0,0584 & 0,1671 & & & \\
\hline C. León & 0,0616 & 0,2098 & 0,7287 & 0,3217 & 0,3333 & 0,3450 & 422,52 & 58,87 & $-52,65$ \\
\hline Std. Dev. & 0,0140 & 0,0733 & 0,2111 & 0,1165 & 0,0788 & 0,2182 & & & \\
\hline C. Valenciana & 0,0432 & 0,1741 & 0,7827 & 0,2642 & 0,3534 & 0,3824 & 510,95 & 103,01 & $-51,14$ \\
\hline Std. Dev. & 0,0120 & 0,0639 & 0,1838 & 0,1012 & 0,0708 & 0,1835 & & & \\
\hline Extremadura & 0,0393 & 0,2029 & 0,7578 & 0,2989 & 0,3995 & 0,3016 & 660,04 & 96,89 & $-60,20$ \\
\hline Std. Dev. & 0,0066 & 0,0484 & 0,1290 & 0,0723 & 0,0322 & 0,1269 & & & \\
\hline Galicia & 0,0718 & 0,2254 & 0,7028 & 0,3506 & 0,3499 & 0,2995 & 388,16 & 55,24 & $-57,38$ \\
\hline Std. Dev. & 0,0167 & 0,0697 & 0,2171 & 0,1164 & 0,0710 & 0,1795 & & & \\
\hline Madrid & 0,0358 & 0,1687 & 0,7955 & 0,2520 & 0,3652 & 0,3828 & 604,74 & 116,44 & $-51,88$ \\
\hline Std. Dev. & 0,0087 & 0,0589 & 0,1596 & 0,0895 & 0,0581 & 0,1695 & & & \\
\hline Murcia & 0,0369 & 0,1741 & 0,7890 & 0,2626 & 0,4007 & 0,3367 & 611,07 & 130,12 & $-57,32$ \\
\hline Std. Dev. & 0,0129 & 0,0443 & 0,1500 & 0,0785 & 0,0552 & 0,1005 & & & \\
\hline Navarra & 0,0082 & 0,0959 & 0,8958 & 0,1419 & 0,3855 & 0,4726 & 1625,67 & 301,88 & $-47,25$ \\
\hline Std. Dev. & 0,0032 & 0,0222 & 0,2999 & 0,0393 & 0,1104 & 0,2009 & & & \\
\hline País Vasco & 0,0525 & 0,1834 & 0,7641 & 0,2842 & 0,3650 & 0,3509 & 441,18 & 99,00 & $-54,08$ \\
\hline Std. Dev. & 0,0156 & 0,0679 & 0,2130 & 0,1134 & 0,0634 & 0,1560 & & & \\
\hline
\end{tabular}


Gráfico 1. Probabilidad predicha "cuidador/a ocupado/a sin problemas laborales" ( $\mathrm{PL}=1)$

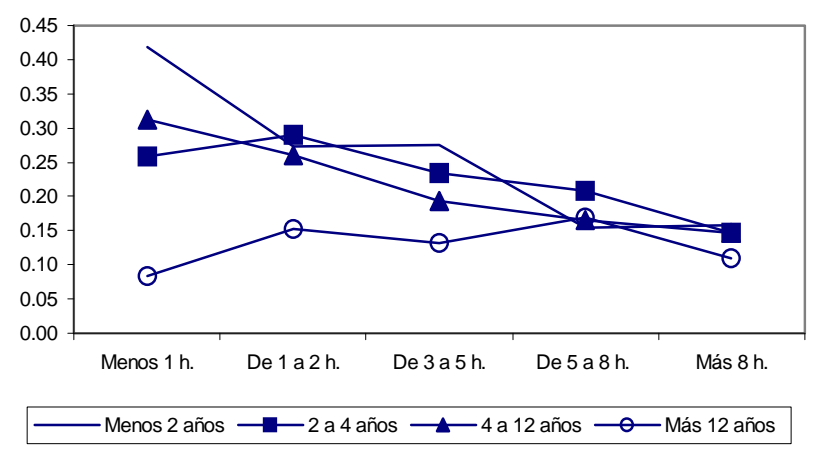

Gráfico 2. Probabilidad predicha para "cuidador/a ocupado/a con problemas laborales" $(\mathrm{PL}=2)$

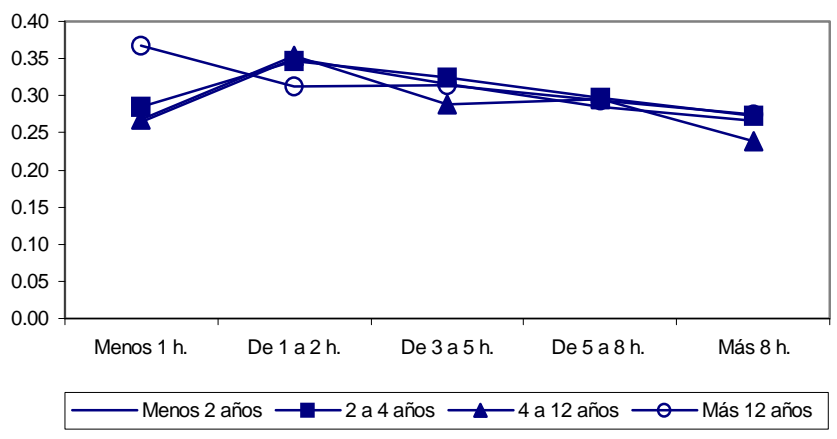

Gráfico 3. Probabilidad predicha para "cuidador/a que se ha visto obligado a dejar de trabajar" $(\mathrm{PL}=3)$

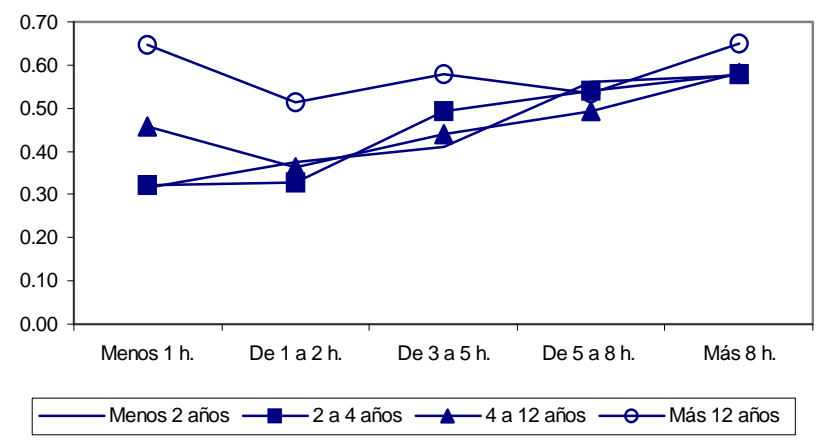

